Pure and Applied Mathematics Quarterly

Volume 1, Number 4

(Special Issue: In Memory of

Armand Borel, Part 3 of 3 )

$939-971,2005$

\title{
Conley Index Theory and Novikov-Morse Theory
}

\author{
Huijun Fan and Jürgen Jost \\ In memory of Armand Borel
}

\begin{abstract}
We derive general Novikov-Morse type inequalities in a Conley type framework for flows carrying cocycles, therefore generalizing our results in [FJ2] derived for integral cocycle. The condition of carrying a cocycle expresses the nontriviality of integrals of that cocycle on flow lines. Gradient-like flows are distinguished from general flows carrying a cocycle by boundedness conditions on these integrals.
\end{abstract}

\section{Introduction}

In the 80's, S. P. Novikov [No1, No2] considered Morse type inequalities relating the zeros of a closed Morse 1-form $\omega$ to the topology of the underlying space $X$.

Let $p$ be a zero point of a closed 1-form $\omega$, then near $p$, one has $\omega=d f_{p}$, where $f_{p}$ is unique up to a constant. The Morse index of $\omega$ at $p$ is defined as the Morse index of $f_{p}$ at $p . p$ is said to be non-degenerate if $f_{p}$ is non-degenerate at $p$. $\omega$ is called a closed Morse 1-form if each zero point $p$ of $\omega$ is non-degenerate. Let $S_{i}(\omega)$ be the set of the zero points of $\omega$ with index $i$. Define

$$
c_{i}:=\# S_{i}(\omega)
$$

Then the Morse type inequalities are

$$
\begin{aligned}
c_{i} & \geq b_{i}([\omega]) \\
\sum_{j=0}^{i}(-1)^{i-j} c_{j} & \geq \sum_{j=0}^{i}(-1)^{i-j} b_{j}([\omega])
\end{aligned}
$$

Received March 26, 2005.

The first author is partially supported by Research Fund for Returned Overseas Chinese Scholars 20010107 and the Partner Group of the Max-Planck-Institute for Mathematics in the Sciences in Leipzig at the Chinese Academy of Sciences. 
for $i=0,1 \cdots, m$. Here the Novikov numbers $b_{i}([\omega])$ are determined only by $X$ and the cohomology class $[\omega]$. These Morse type inequalities involving Novikov numbers are called Novikov inequalities.

Novikov inequalities were obtained by constructing the Novikov complex $\left(C_{*}, \partial\right)$ with respect to the closed Morse 1-form $\omega$. If $[\omega]$ is an integral cohomology class, then each $C_{i}$ is a free $\mathbb{Z}((t))$-module generated by the set of all the zeros of $\omega$ with index $i$. Here $\mathbb{Z}((t)):=\mathbb{Z}[[t]]\left[t^{-1}\right]$ is the Novikov ring. For the detailed construction of the boundary operators and some of their properties, see the discussion in $[\mathrm{BF}],[\mathrm{Pa}],[\mathrm{Ra}]$ and the references there.

M. Farber and A.A. Ranicki [FR] have used a noncommutative localization method to construct a universal complex for a rank 1 Morse closed 1-form. Subsequently, M. Farber [Fa2] applied the method to the general case, which can induce many kinds of Novikov complexes. In a series of papers [Fo1, Fo2, Fo3], R. Forman developed the discrete Morse theory and Novikov theory in a combinatorial category. Those Novikov-Morse inequalities are proved also by constructing certain combinatory Morse(Novikov) complexes, e.g., using the Witten deformation technique. In order to use the techniques from the smooth category, he introduced combinatorial vector fields and differential forms.

Novikov theory for closed 1-forms is very important, since many functionals, such as the symplectic action functional, the Chern-Simons functional and many Hamiltonian systems from electromagnetism and fluid mechanics are multi-valued functionals. Though these are multi-valued functionals in infinite dimensional spaces, people hope that the Novikov theory in finite dimension can provide a model for the infinite dimensional case, and that it can provide a strong topological method to find the critical points of the multi-valued functionals.

Though Novikov theory for closed 1-forms has been studied for many years, the various proofs of Novikov inequalities were all based on constructing various (analytic or topological )Novikov complexes. Hence the closed 1-form needs some non-degeneracy assumptions. One could not handle the case if the closed 1-form $\omega$ has general degenerate zero points (zero locus). On the other hand, the behavior of the dynamical systems generated by a closed 1-form has not been carefully studied. For instance, the previous research was limited to the smooth category. Though there should be an analogue in the continuous category (i.e., for continuous dynamical systems), if so, then comes the natural question, what is the relation between this analogue and the well-known gradient-like dynamical systems?

In [FJ2], we introduced a concept of "flow carrying a cocycle $\alpha$ ( $\alpha$-flow and generalized $\alpha$-flow)", where $\alpha$ is a 1-dimensional cocycle in the bounded AlexanderSpanier cohomology (see i.e., $[\mathrm{Sp}]$ ). This is the analogue of the flow generated by 
a closed 1-form. Actually, as shown in [FJ2], (generalized) $\alpha$-flows characterize most important dynamical systems, in particular the following:

- The gradient flow of a Morse function $f$ is a $d f$-flow.

- The gradient flow of a closed Morse 1-form $\omega$ is an $\omega$-flow and certain perturbation flows are also $\omega$-flows.

- If an $\alpha$-flow has no fixed point, then this flow is a flow "carrying a cohomology class" as introduced in [Ch].

- An $\alpha$-Morse-Smale flow as defined in [FJ2] which is a generalization of the famous Morse-Smale flow; the generalization here essentially consists in allowing the existence of "cycles"

- If $\alpha$ is a coboundary, then this flow is a gradient-like flow. Conversely, if a flow is a gradient-like flow, then this flow carries a coboundary $\delta g$ where $g$ is a Lyapunov function.

A flow carrying a cocycle $\alpha$ has a $\pi$-Morse decomposition (Theorem 3.4.4 in [FJ2]). If $\alpha$ is an integral cocycle, we can obtain the Novikov-Morse type inequalities, Theorem 4.4.1 in [FJ2]. This theorem uses the Conley index to get the "local" topological information of the isolated invariant sets.

Those inequalities in [FJ2] are a generalization of the Novikov inequalities for an integral closed Morse 1-form. Starting from these inequalities, we can recover many Novikov type inequalities found before. For example, we can recover the Novikov inequalities for an integral closed 1-form $\omega$ having Bott type nondegenerate zero sets, which was given in [Fa2]. In addition, our theory has some new features:

- Novikov inequalities now hold in the continuous category (i.e.for (generalized) $\alpha$-flows).

- New Novikov inequalities (Theorem 4.4.1 in [FJ2]) for a general closed 1form without any non-degeneracy requirement (However we have to point out here that we need some extra regularity assumption to the zero locus of the closed 1-form in Theorem 4.4.1 in [FJ2], which will be shown in this paper).

- New Novikov inequalities for $\alpha$-Morse Smale flows.

- Vanishing of the Novikov numbers $b_{i}([\alpha])$, if the manifold allows the existence of a flow carrying a cohomology class $\alpha$.

However, [FJ2] is mainly focused on the explanation of the flow carrying a cocycle $\alpha$, and on the proof of the Novikov-Morse inequalities for this flow under the assumption that $\alpha$ is an integral cocycle.

This paper is the second one. There are two aims. The first aim is to give a sufficient and necessary description of the relation between $\alpha$-flows and the gradient-like flows. This will be given in section 3 . The second aim is to generalize 
the Novikov-Morse inequalities for integral cocycles $\alpha$ to the analogous results for higher rank cocycles $\alpha$. This is discussed in sections 4,5. To do this, one should prove an analogue of Theorem 4.2.1 in [FJ2]. This is a nontrivial generalization, since the exit set of the lifting flow becomes more complicated when compared with the integral cocycle case. One should handle carefully the deformation complex in the corners. As long as we have the analogous theorem, we can easily generalize those theorems in [FJ2] by following proofs in [FJ2].

In section 2, we recollect some basic definitions and examples of flows carrying a cocycle $\alpha$.

Recently, M. Farber [Fa3](2001) also proposed the concept:"Lyapunov oneform" $\omega$ for a pair $(\Phi, Y)$, where $\Phi$ is a continuous flow and $Y$ is a closed invariant set in $\Phi$. Since a flow $\Phi$ allowing the existence of a Lyapunov one-form $\omega$ is actually a flow carrying a cocycle $\omega$ introduced in [FJ2], our definition seems more general than his. In [Fa3, Fa4], Farber constructed the corresponding LjusternikSchnirelman theory. In [FKLZ], the authors discussed the existence of "Lyapunov one-forms".

The results presented in this paper were obtained in 2000 and circulated in preliminary preprint form in [FJ1].

\section{Review of flows carrying a cocycle}

In [FJ2], we have defined the dynamical systems, flows carrying a cocycle. In this section we will recollect some facts about such dynamical systems.

Let $v$ be a flow defined on the compact metric space $(X, d)$ with metric $d$. We always assume in this paper that $\mathcal{A}$ is an isolated invariant set of $v$ having finitely many connected components. Denote its connected components by $A_{i}, i=1,2, \cdots, n$.

Let $A_{i} \subset \mathcal{A}$ be a component with an isolating neighborhood $U_{i}$, then for any given closed neighborhood $V_{i}$ of $A_{i}$ in $U_{i}$, we can define the thickening stable and unstable sets of $A_{i}$ in $V_{i}$ as follows:

$A_{i}^{+}\left(V_{i}, r\right):=\left\{x \in V_{i} \mid\right.$ there is a $t \geq 0 . s . t . x \cdot[0, t] \subset V_{i}$ and $\left.x \cdot[0, t] \cap \operatorname{cl}\left(B_{r}\left(A_{i}\right)\right) \neq \emptyset\right\}$

$A_{i}^{-}\left(V_{i}, r\right):=\left\{x \in V_{i} \mid\right.$ there is a $t \leq 0$. s.t.x $\cdot[t, 0] \subset V_{i}$ and $\left.x \cdot[t, 0] \cap \operatorname{cl}\left(B_{r}\left(A_{i}\right)\right) \neq \emptyset\right\}$.

The compact set $A_{i}\left(V_{i}, r\right):=A_{i}^{+}\left(V_{i}, r\right) \cup A_{i}^{-}\left(V_{i}, r\right)$ becomes an isolating neighborhood of $A_{i}$, which is called the flow neighborhood of $A_{i}$. If $r$ is small enough, then $\left(A_{i}^{-}\left(V_{i}, r\right), A_{i}\left(V_{i}, r\right)\right)$ forms an index pair for $A_{i}$ (see [FJ2] for detail). Sometimes we will denote the flow neighborhood $A_{i}\left(V_{i}, r\right)$ simply by $A_{i}(r)$ if we don't emphasize the neighborhood $V_{i}$ of $A_{i}$.

When restricted to the open space $X \backslash \cup_{i=1}^{n} A_{i}(r)$, all the connected trajectories of the flow $v$ can be classified into three types: 
(1) trajectories with domain $(a, b),-\infty<a<b<+\infty$;

(2) trajectories with domain $(a,+\infty)$ or $(-\infty, b),-\infty<a, b<+\infty$;

(3) $(-\infty,+\infty)$.

We denote the sets of three type trajectories by $\Gamma_{1}^{\mathcal{A}}(r), \Gamma_{2}^{\mathcal{A}}(r)$ and $\Gamma_{3}^{\mathcal{A}}(r)$, respectively. If $A_{i}$ is a point $p_{i}$ in $X$ for $i=1, \cdots, n$, then we denote $\Gamma_{\gamma}^{\mathcal{A}}(r)$ by $\Gamma_{\gamma}(r), \gamma=1,2,3$.

In [FJ2], we have defined the continuous 1-cocycle $\alpha$ in bounded AlexanderSpanier cohomology theory and its integration along a chain (the integration of 1-cocycle along a curve was already defined in $[\mathrm{Ch}]$ ).

If $\left.[\alpha]\right|_{A}=0$ for a closed set $A \subset X$, then by the continuity of Alexander-Spanier cohomology theory there is a continuous function $\beta$ such that $\alpha=\delta \beta$. Define $I_{\alpha}(x, y):=\beta(y)-\beta(x)$, for $x, y \in A$. It is obvious that $I_{\alpha}(x, y)$ depends only on $[\alpha]$.

Definition 2.1 Let $\alpha$ be a continuous 1-cocycle on the compact metric space $(X, d)$. The flow $v$ defined on $X$ is said to be a generalized $\alpha$-flow with respect to an isolated invariant set $\mathcal{A}$ consisting of connected components $\left\{A_{n}, \cdots, A_{1}\right\}$, if there exist a small $r>0$ and $a T_{0}>0$ such that for some $\rho>0$ and $0 \leq \lambda<1$, the following conditions are satisfied:

(1) $\left.[\alpha]\right|_{A_{i}}=0, \max _{(x, y) \in A_{i}(r) \times A_{i}(r)}\left|I_{\alpha}(x, y)\right| \leq \lambda \rho$, for $1 \leq i \leq n$.

(2) for any trajectory $\gamma \in \Gamma_{1}^{\mathcal{A}}(r)$,

$$
\int_{\gamma} \alpha \geq \rho
$$

(3) if $\gamma^{T_{0}}$ denotes any sub-trajectory of $\gamma \in \Gamma_{2}^{\mathcal{A}}(r) \cup \Gamma_{3}^{\mathcal{A}}(r)$ with time interval $T_{0}$, then

$$
\int_{\gamma^{T_{0}}} \alpha \geq \rho
$$

Remark 2.1 In fact, we can take the constant $\rho$ in condition (3) to be different from the one in conditions (1) and (2). This broader assumption will not change any proofs and conclusions in [FJ2]. Since $\alpha$ is a continuous cocycle, the three conditions in Definition 2.1 also hold for $r^{\prime}$ sufficiently close to $r$.

Definition 2.2 If the set $\mathcal{A}$ in the definition of a generalized $\alpha$-flow $v$ consists only of points, then $v$ is called an $\alpha$-flow. If $v$ is an $\alpha$-flow or generalized $\alpha$-flow, we simply call it a flow carrying a cocycle $\alpha$.

Some interesting and important (generalized) $\alpha$-flows are given below, more examples can be found in [FJ2]. 
Example 2.1 In classical Novikov theory, the closed Morse 1-forms or the Botttype closed 1-form were considered frequently. Now in the present example, we consider a closed 1-form $\omega$ with no Bott-type non-degenerate restriction to its zero locus $\mathcal{A}$. We only assume that each connected component $A_{i} \subset \mathcal{A}, i=1, \cdots, n$, is a Lipschitz submanifold. So each $A_{i}$ has naturally a tubular neighborhood $U_{i}$ such that $A_{i}$ is the deformation retract of $U_{i}$.

Let $V$ be the flow generated by the closed 1-form $\omega$ and a Riemannian structure. We want to show that $V$ is a generalized $\omega$-flow w.r.t. its zero locus $\mathcal{A}$.

Firstly we show that any $A \in \mathcal{A}$ is an isolated invariant set. Let $U$ be a tubular neighborhood of $A$ and $\pi: U \rightarrow A$ be the projection. We want to show $I(U)=A$. Let $p \in I(U)$, then for any $t_{0}, t_{1}, t_{0}<t_{1}$,

$$
\begin{aligned}
& \int_{t_{0}}^{t_{1}}|\omega(p \cdot t)|^{2} d t=\int_{\left[p \cdot t_{0}, p \cdot t_{1}\right]} \omega=\int_{\left[p \cdot t_{0}, \pi\left(p \cdot t_{0}\right)\right]} \omega \\
& +\int_{\left[\pi\left(p \cdot t_{0}\right), \pi\left(p \cdot t_{1}\right)\right]} \omega+\int_{\left[\pi\left(p \cdot t_{1}\right), p \cdot t_{1}\right]} \omega \\
& \leq C
\end{aligned}
$$

We use the facts that the integration of $\omega$ is only dependent on the relative homotopy class of $\left[p \cdot t_{0}, p \cdot t_{1}\right]$ and the integration $\int_{\gamma} w$ is bounded from above by a constant depending on the length of the Lipschitz continuous curve $\gamma$.

The above inequality implies that there exist sequences $t_{0 k} \rightarrow-\infty$ and $t_{1 k} \rightarrow$ $+\infty$ such that $\left|\omega\left(p \cdot t_{0 k}\right)\right| \rightarrow 0$ and $\left|\omega\left(p \cdot t_{1 k}\right)\right| \rightarrow 0$ as $k \rightarrow \infty$. Now the above formula forces $|\omega(p \cdot t)|=0, \forall t \in \mathbb{R}$. This proves that $p \in A$.

From the above conclusion, we can take a closed neighborhood $V_{i}$ of $A_{i}$ contained in an isolating neighborhood $U_{i}$ s.t. $d\left(\partial V_{i}, \partial U_{i}\right)>r_{0}, \min _{x \in \overline{U_{i}-V_{i}}}|\omega(x)| \geq$ $\varepsilon_{0}$. Now take the flow neighborhood $A_{i}(r):=A_{i}\left(B_{2 r}\left(A_{i}\right), r\right)$ for small $r$. Since any $\gamma \in \Gamma_{1}^{\mathcal{A}}(r)$ has to go across $U_{i}-V_{i}$ for some $i$, we have

$$
\begin{aligned}
\int_{\gamma} \omega & =\int_{a}^{b}|\omega(\gamma(t))|^{2} d t \\
& \geq \min _{1 \leq i \leq n}\left(\min _{x \in \overline{U_{i}-V_{i}}}|\omega(x)| \cdot d\left(\partial V_{i}, \partial U_{i}\right)\right) \geq r_{0} \varepsilon_{0}
\end{aligned}
$$

Now if we take $r$ sufficiently small, then (1) and (2) in Definition 2.1 holds.

To prove (3) in Definition 2.1, we fix $r$ and then choose $T_{0}$ sufficiently large such that $\forall \gamma \in \Gamma_{2}^{\mathcal{A}}(r) \cup \Gamma_{3}^{\mathcal{A}}(r)$,

$$
\int_{\gamma\left(T_{0}\right)} \omega=\int|\omega(\gamma(t))|^{2} d t \geq \min _{x \in X-B_{r}\left(A_{i}\right)}|\omega(x)|^{2} T_{0} .
$$

Therefore we have proved that $V$ is a generalized $\omega$-flow w.r.t. its zero locus. As a direct corollary, the flow generated by a closed 1-form with Bott-type nondegenerate zero sets is a generalized $\omega$-flow w.r.t. its zero locus. 
Example 2.2 A flow $v$ on a manifold $M$ is called a Morse-Smale flow (after $[\mathrm{Sm}])$ if it satisfies:

(1) The chain recurrent set of $v$ consists of a finite number of hyperbolic closed orbits and hyperbolic fixed points .

(2) The unstable manifold of any closed orbit or fixed point has transversal intersection with the stable manifold of any closed orbit or fixed point.

Smale $[\mathrm{Sm}]$ proved that such flows have "global" gradient-like structures and have a Morse decomposition which induces the Morse inequalities.

However, in some cases, although the nonwandering set of the flow contains only the hyperbolic periodic orbits and the hyperbolic fixed points, the flow is not a Morse-Smale flow because of the existence of "cycles" which consist of some orbits "connecting" different invariant sets and form a closed curve. We can give a definition of such flows when restricted to the category of flows carrying a cocycle.

$\alpha$-Morse-Smale flow Let $v$ be a generalized $\alpha$-flow with respect to an isolated invariant set $\mathcal{A}=\left\{A_{n}, \cdots, A_{1}\right\}$. If $\mathcal{A}$ contains only the hyperbolic orbits or hyperbolic fixed points, then $v$ is called an $\alpha$-Morse-Smale flow.

Example 3.3.9 in [FJ2] provides concrete $\alpha$-Morse-Smale flows.

Example 2.3 (Flows carrying a cohomology class) We consider an extreme case namely that $\mathcal{A}$ is empty in the definition of an $\alpha$-flow $v$. In this case, the set $\Gamma_{1}(r) \cup \Gamma_{2}(r)=\emptyset$ and the only condition that makes $v$ an $\alpha$-flow is that there exist constants $\rho>0$ and $T_{0}>0$ such that for any trajectory $\gamma\left(T_{0}\right)$ with time interval $T_{0}$, we have

$$
\int_{\gamma\left(T_{0}\right)} \alpha \geq \rho
$$

Now the $\alpha$-flow $v$ becomes a so called "flow carrying a cohomology class" as introduced by R.C.Churchill $[\mathrm{Ch}]$. The reason that the flow is called "carrying a cohomology class" is that the above condition is independent of the choice of the representative in the cohomology class $[\alpha]$. In fact, if $\alpha_{1} \in[\alpha]$ is another cocycle, then there exists a coboundary $\delta \beta$, such that

$$
\alpha_{1}-\alpha \simeq \delta \beta
$$

and so

$$
\begin{aligned}
\int_{\gamma\left(k T_{0}\right)} \alpha_{1} & =\int_{\gamma\left(k T_{0}\right)} \alpha+\beta\left(e\left(\gamma\left(k T_{0}\right)\right)\right)-\beta\left(s\left(\gamma\left(k T_{0}\right)\right)\right) \\
& \geq k \rho-2 M_{\beta}
\end{aligned}
$$


where $s(\gamma)$ and $e(\gamma)$ are the start point and the end point of the trajectory $\gamma$, and $M_{\beta}$ is the bound of $\beta$. Hence if we choose $k>\left[\frac{2 M_{\beta}+\rho}{\rho}\right]+1$, then we have

$$
\int_{\gamma\left(k T_{0}\right)} \alpha_{1} \geq \rho
$$

The existence of a flow carrying a cohomology class in a manifold will induce the vanishing of the Novikov numbers. This result will be given in the last section.

\section{3. $\alpha$-flows and gradient-like flows}

After defining the $\alpha$-flows, it is natural to find the relation between $\alpha$-flows and the well-known gradient-like flows. It seems that an $\alpha$-flow for $\alpha$ being a nontrivial cocycle should be a nongradient-like flow. However the following simple example shows that this is not true.

Example 3.1 Let $S^{1}$ be the unit circle with the standard metric. Let $\varphi_{1}$ and $\varphi_{2}$ be two strictly monotonically increasing functions in the interval $[0, \pi]$ satisfying the following conditions

$$
\begin{aligned}
& \varphi_{1}(0)=0, \varphi_{1}^{\prime}(0)=0 ; \varphi_{1}(\pi)=3 \pi, \varphi_{1}^{\prime}(\pi)=0 \\
& \varphi_{2}(0)=0, \varphi_{2}^{\prime}(0)=0 ; \varphi_{2}(\pi)=\pi, \varphi_{2}^{\prime}(\pi)=0 .
\end{aligned}
$$

Define a $C^{1}$-smooth circle-valued function

$$
f(x)= \begin{cases}e^{i \varphi_{1}(x)} & 0 \leq x \leq \pi \\ e^{i \varphi_{2}(2 \pi-x)} & \pi \leq x \leq 2 \pi\end{cases}
$$

Consequently, $f(x)$ induces a cocycle $\alpha(x, y):=J(f)(x, y): S^{1} \times S^{1} \rightarrow \mathbb{R}$ such that if $y$ lies in a small neighborhood of $x$, then

$$
\int_{[x, y]} \alpha=\arg f(y)-\arg f(x) .
$$

Here the map $J$ is defined in proposition 3.1.1 of [FJ2]. This cocycle is a nontrivial cocycle. Since if we let $\gamma$ be the oriented curve starting from the point $\theta=0$ and then going around the circle in the anticlockwise direction, then for any integer $l$, we have

$$
\int_{l \gamma} \alpha=2 \pi l
$$

Now we consider the gradient-like flow $v$ on $S^{1}$ that has two fixed points at $\theta=0, \pi$, and flows from the point $\theta=0$ to the point $\theta=\pi$. Then it is easy to check that $v$ carries the cocycle $\alpha$, if we let $r$ sufficiently small and take the parameters $\lambda=1 / 2, \rho=1$ and $\Gamma_{2}(r) \cup \Gamma_{3}(r)=\emptyset$ in the definition 2.1.

Thus, this example demonstrates that a gradient-like flow can carry a nontrivial cocycle. 
Remark 3.1 It is easy to see that for any $x \in S^{1}$, and $t>0, \int_{[x, x \cdot t]} \alpha>0$. Hence the pull-back form $f^{*}(d \theta)$ is a Lyapunov 1-form for the pair $(-v, \theta=0, \pi)$, which is defined in [Fa3, Fa4]. Though the smooth closed 1-form $f^{*}(d \theta)$ is a nontrivial smooth closed 1 -form, $-v$ is a gradient-like flow.

We will give a series of theorems and examples to formulate the relation between $\alpha$-flows and gradient-like flows. The following theorem is already given in [FJ2].

Theorem 3.1 Let $v$ be an $\alpha$-flow on the compact metric space $(X, d)$. If $\alpha$ is a trivial cocycle, then $v$ is a gradient-like flow. Conversely, if $v$ is a gradientlike flow, then $v$ is a $\delta g$-flow for some continuous function $g$.

We will give necessary and sufficient conditions to distinguish $\alpha$-flows and gradientlike flows.

In this part we assume that $v$ is a gradient-like flow with finitely many fixed points $\left\{p_{j} ; j=1,2, \cdots, n\right\}$. We can choose an associated Lyapunov function $g$ and $\epsilon_{0}>0$ such that for small $r$, there are $\operatorname{dist}\left(g\left(B_{r}\left(p_{j}\right)\right), g\left(B_{r}\left(p_{i}\right)\right)\right) \geq \epsilon_{0}$ for $i \neq j$, and $\operatorname{osc}_{B_{r}(x)} g(y) \leq \frac{\epsilon_{0}}{4}$ for any $x$. Firstly we study the local structure of the gradient-like flow $v$.

Local structure of gradient-like flows Let $p$ be a fixed point of the gradientlike flow $v$. Let $0<s<\frac{r}{2}$ and let $B_{\frac{r}{2}}(p)$ be a closed ball centered at $p$ with radius $\frac{r}{2}$. Define two sets on the sphere $\partial B_{r}(p)$,

$$
\begin{aligned}
& B_{r, s}^{+}(p)=\left\{x \in \partial B_{r}(p) \mid[x, x \cdot t] \cap \partial B_{s}(p) \neq \emptyset \text { for some } t>0\right\} \\
& B_{r, s}^{-}(p)=\left\{x \in \partial B_{r}(p) \mid[x \cdot t, x] \cap \partial B_{s}(p) \neq \emptyset \text { for some } t<0\right\}
\end{aligned}
$$

For any point $x \in B_{r, s}^{+}(p)$, let $t_{x}$ denotes the reach time of the trajectory $[x, x \cdot t](t>0)$ to the sphere $\partial B_{\frac{r}{2}}(p)$. We have the following lemma.

Lemma 3.1 There exists a $r_{0}>0$, such that $\forall r \in\left(0, r_{0}\right)$ the following hold: (1) $B_{r, s}^{ \pm}(p)$ are closed sets on $\partial B_{r}(p)$.

(2) $t_{x}$ is a lower semicontinuous function on $B_{r, s}^{+}(p)$.

Proof. $B_{r, s}^{ \pm}(p)$ are actually the boundaries of the flow neighborhoods $A^{ \pm}\left(B_{r}, s\right)$ defined at the beginning of section 2 (or see [FJ2] for detail). Hence they are closed if $s$ is small enough. Here we prove that it is true for $s<r / 2$.

To prove (1), we need only to prove that the set $\partial B_{r}(p)-B_{r, s}^{+}(p)$ is open in $\partial B_{r}(p)$. Since any $x \in \partial B_{r}(p)-B_{r, s}^{+}(p)$ will finally drop into another fixed point 
$q$, by the choice of $g(x)$, we can choose $a<b$ satisfying for smaller $r$

$$
B_{r}(p) \subset g^{-1}([b,+\infty]) ; B_{r}(q) \subset g^{-1}((-\infty, a])
$$

Since $x \cdot t(t>0)$ drops into $q$, there exists a $T_{x}>0$ such that $x \cdot T_{x}$ is in the interior of $B_{r}(q)$. Therefore there is a closed ball $D_{\delta}(x)$ such that for any $y \in D_{\delta}(x) \cap \partial B_{r}(p), y \cdot T_{x}$ is in the interior of $B_{r}(q)$. We can choose $\delta$ small enough such that the set $D_{\delta}(x) \cdot\left[0, T_{x}\right] \cap \partial B_{s}(p)=\emptyset$. Since $y \cdot T_{x} \in g^{-1}((-\infty, a]), y$. $\left[T_{x},+\infty\right) \in g^{-1}((-\infty, a])$. Therefore $D_{\delta}(x) \cdot[0,+\infty] \cap \partial B_{s}(p)=\emptyset$ in view of (3.1). This shows that $\partial B_{r}(p)-B_{r, s}^{+}(p)$ is open in $\partial B_{r}(p)$.

To prove (2), let $x \in B_{r, s}^{+}(p)$ and let $t_{x}<+\infty$ be the arrival time. We need only to prove that for any small $\epsilon>0$, there exists a neighborhood $D_{\delta}(x)$ of $x$ such that for any $y \in D_{\delta}(x) \cap B_{r, s}^{+}(p)$,

$$
t_{y} \geq t_{x}-\epsilon
$$

Since $t_{x}$ is the arrival time of the trajectory $x \cdot t(t>0)$ to $\partial B_{\frac{r}{2}}(p), x \cdot\left[0, t_{x}-\epsilon\right]$ has a positive distance to $\partial B_{\frac{r}{2}}(p)$. By the continuity of the flow $v$, there is a neighborhood $D_{x}$ such that $\bar{D}_{x}^{2} \cdot\left[0, t_{x}-\epsilon\right]$ has a positive distance to $\partial B_{\frac{r}{2}}(p)$ as well. Hence for $y \in D_{x} \cap B_{r, s}^{+}(p),(3.2)$ is true.

Since the Lyapunov function $g(x)$ is strictly decreased along any nonconstant trajectory, there exists a $\delta_{x}>0$ such that $g(x)-g\left(x \cdot t_{x}\right)=2 \delta_{x}$. By lemma $3.1,(2)$ and the property of $g(x)$, the function $g(y)-g\left(y \cdot t_{y}\right)$ is lower semicontinuous with respect to $y$ on $B_{r, s}^{+}(p)$. Hence $\delta_{r, s}^{+}(p):=\min _{x \in B_{r, s}^{+}(p)} \delta_{x}$ is positive and $\forall x \in B_{r, s}^{+}(p), g(x)-g\left(x \cdot t_{x}\right)>\delta_{r, s}^{+}(p)$. In the same way, we can obtain a $\delta_{r, s}^{-}(p)>0$ such that $\forall x \in B_{r, s}^{-}(p)$, there is $g\left(x \cdot\left(-t_{x}\right)\right)-g(x)>\delta_{r, s}^{-}(p)$.

Let $0<s_{1}<s_{2}<\frac{r}{2}$, then we have the following conclusions:

(1) $B_{r, s_{1}}^{+}(p) \subset B_{r, s_{2}}^{+}(p) ; B_{r, s_{1}}^{-}(p) \subset B_{r, s_{2}}^{-}(p)$

(2) $\delta_{r, s_{2}}^{ \pm}(p) \leq \delta_{r, s_{1}}^{ \pm}(p)$

(3) There exists $s_{0}>0$, such that for any $0<s<s_{0}, B_{r, s}^{+}(p) \cap B_{r, s}^{-}(p)=\emptyset$

The first two conclusions are obvious. For (3), we can choose $s_{0}>0$ satisfying $\operatorname{osc}_{x \in B_{s_{0}}(p)} g(x)<\min \delta_{r, s_{0}}^{ \pm}(p)$. If there is a $s \in\left(0, s_{0}\right)$ such that $B_{r, s}^{+}(p) \cap B_{r, s}^{-}(p) \neq$ $\emptyset$, then this means that there is a trajectory in $\bar{B}_{r}(p)$ which has non empty intersection with $\partial B_{r}(p)$ and $\partial B_{s}(p)$. But this is absurd, because $g(x)$ is strictly decreacing along any trajectory. After travelling from $\partial B_{s}(p)$ to $\partial B_{r}(p)$, then back to $\partial B_{s}(p)$, the value of $g(x)$ will decrease at least $\delta_{r, s}^{+}(p)+\delta_{r, s}^{-}(p)$ which contradicts the fact that $\operatorname{osc}_{x \in B_{s}(p)} g(x) \leq \operatorname{osc}_{x \in B_{s_{0}}(p)} g(x)<\min \delta_{r, s}^{ \pm}(p)$. Hence for $0<s<s_{0}$, (3) holds. 
Integration of cocycles in a gradient-like flow Let $\alpha$ be a cocycle on $X$. Then for sufficiently small $r$, there is an associated $r$-covering $\mathcal{U}(r)$ (covering consisting of radius $r$-balls) such that when restricted to the closure of each open ball in $\mathcal{U}(r), \alpha$ is a coboundary $\delta \beta$, where $\beta$ is a bounded function.

Define $\delta_{0}(r)=\min _{1 \leq j \leq n} \delta_{r, \frac{r}{4}}^{ \pm}\left(p_{j}\right)$. Since $g(x)$ is uniformly continuous, there exists $s_{0} \leq \frac{r}{4}$ satisfying $\operatorname{osc}_{B_{s_{0}}(x)} g(y)<\frac{1}{3} \delta_{0}(r)$ for any $x \in X$. By our choice and in view of the analysis of the local structure of gradient-like flows, it is easy to see that the following inequality holds:

$$
3 \max _{x} \operatorname{osc}_{y \in B_{s_{0}}(x)} g(y)<\delta_{0}(r)<\delta_{r, \frac{r}{4}}^{ \pm}\left(p_{j}\right) \leq \delta_{r, s_{0}}^{ \pm}\left(p_{j}\right)
$$

for $j=1,2, \cdots, n$.

Theorem 3.2 Let $v$ be a gradient-like flow with finitely many fixed points. $\alpha$ is a cocycle with an associated $r$-covering. Let $\mathcal{U}(s)$ be an $s$-covering with $s<s_{0}<\frac{r}{4}$ for some $s_{0}$ satisfying (3.3). Then there exist $M>0$ and $T\left(s_{0}\right)>0$ depending on $r, n, \alpha$ but not on such that for any $\left(\mathcal{U}(s), T\left(s_{0}\right)\right)$-chain $\tilde{\gamma}$, we have

$$
\left|\int_{\tilde{\gamma}} \alpha\right| \leq M
$$

Recall that a $(\mathcal{U}(s), T)$-chain from $x$ to $x^{\prime}$ is a sequence $\left\{x=x_{1}, \cdots, x_{n+1}=\right.$ $\left.x^{\prime} \mid t_{1}, \cdots, t_{n}\right\}$ such that $t_{i} \geq T$ and each pair $\left(x_{i} \cdot t_{i}, x_{i+1}\right)(i=1, \cdots, n)$ belongs to a ball with radius $s$ in the covering $\mathcal{U}$.

Proof Firstly we will prove that given any $s \leq r$, for any trajectory $\gamma \in \Gamma_{1}(s)$ with domain $[a, b]$, there exists a constant $T(s)$ satisfying $b-a \leq T(s)$.

Let $x \in \partial B_{s}\left(p_{j}\right)$, then either the trajectory $x \cdot t$ or $x \cdot(-t)$ for $t \geq 0$ will flow into some different fixed point of $v$. (There does not exist any trajectory joining one point to itself.) Without loss of generality, we assume that $(x \cdot t)(t \geq 0)$ flows into the point $p_{k}$ for $k \neq j$. (It is possible that the trajectory may pass through some ball $B_{s}\left(p_{l}\right)$, but this does not influence the result below.) Hence there exists a $T_{x}>0$ such that $x \cdot T_{x} \cap B_{\frac{s}{2}}\left(p_{k}\right) \neq \emptyset$. By the continuity of the flow, there exists a small closed ball $D_{s_{x}}(x)$ which satisfies that for any point $y \in D_{s_{x}}(x) \cap \partial B_{s}\left(p_{j}\right)$, the trajectory $\left[y, y \cdot T_{x}\right] \cap \partial B_{s}\left(p_{k}\right) \neq \emptyset$. This shows that for any trajectories starting from $D_{s_{x}}(x) \cap \partial B_{s}\left(p_{j}\right)$ their time intervals are not greater than $T_{x}$. Covering the sphere $\partial B_{s}\left(p_{j}\right)$ by finitely such small closed balls, then it is easy to see that any trajectory in $\Gamma_{1}(s)$ starting from $\partial B_{s}\left(p_{j}\right)$ has time interval not greater than a constant $T_{j}(s)$. Let $T(s)=\max _{1<j<n} T_{j}(s)$, then all the trajectories in $\Gamma_{1}(s)$ have time interval not greater than $T(s)$.

Let $\gamma=\left\{x_{0}, y_{0}, x_{1}, y_{1}, \cdots, x_{k-1}, y_{k-1}, x_{k} \mid t_{0}, \tau_{0}, \cdots, t_{k-1}, \tau_{k-1}\right\}$ be a chain. It 


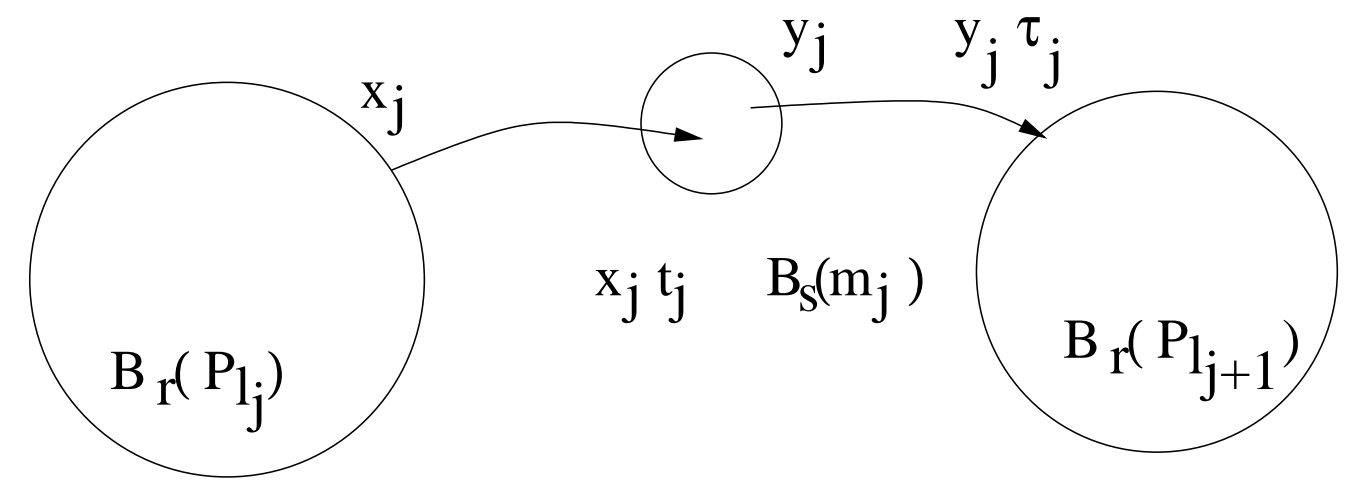

$$
\left(\mathrm{r}, \mathrm{s}_{0}\right) \text {-chain }
$$

is called an $\left(r, s_{0}\right)$-chain if it satisfies the following conditions:

(1): $x_{j} \in \partial B_{r}\left(p_{l_{j}}\right)$ for $j=0,1, \cdots, k-1$ and $x_{k} \in B_{r}\left(p_{l_{k}}\right)$;

(2): $y_{j} \cdot \tau_{j} \in \partial B_{r}\left(p_{l_{j+1}}\right)$ for $j=0,1, \cdots, k-1$;

(3): the point pair $\left(x_{j} \cdot t_{j}, y_{j}\right) \in B_{s}\left(m_{j}\right)$, for $j=0,1, \cdots, k-1$, where those $B_{s}\left(m_{j}\right)^{\prime} s$ are elements in the $s$-covering $\mathcal{U}(s)$ of $X$.

(4): $B_{s}\left(p_{l_{j}}\right), j=0,1, \cdots, k-1$ are $k$ different balls, hence $k \leq n$.

(5): $t_{j}, \tau_{j} \leq T\left(s_{0}\right)$, where $T\left(s_{0}\right)$ is an upper bound for the time interval of all the trajectories in $\Gamma_{1}\left(s_{0}\right)$.

Now compute the integral of $\alpha$ along a $\left(r, s_{0}\right)$-chain $\gamma$.

$$
\begin{aligned}
\left|\int_{\bar{\gamma}} \alpha\right| & \leq \sum_{j=0}^{k-1}\left|\int_{\left[x_{j}, x_{j} \cdot t_{j}\right]} \alpha\right|+\sum_{j=0}^{k-1}\left|\int_{\left[y_{j}, y_{j} \cdot \tau_{j}\right]} \alpha\right| \\
& +\sum_{j=0}^{k-1}\left|\int_{\left[x_{j} \cdot t_{j}, y_{j}\right]} \alpha\right|+\sum_{j=0}^{k-1} \operatorname{osc}_{x \in B_{r}\left(p_{l_{j+1}}\right)} \beta_{p_{l_{j+1}}} \\
& =I+\mathbb{I I}+\mathbb{I I I}+N
\end{aligned}
$$

Since $0<t_{j}, \tau_{j} \leq T\left(s_{0}\right)$, applying corollary 3.2 .3 in [FJ2],

$$
I+\text { II } \leq 2 k C_{1}
$$

where $C_{1}$ is a constant depending only on $\max |\dot{v}(t)|, \alpha$ and $r$.

Connect the point pair $\left(x_{j} \cdot t_{j}, y_{j}\right)$ with a line segment, then its length is at most $2 s$. Using proposition 3.2.2 in [FJ2], we have

$$
\text { III } \leq k C_{2}
$$




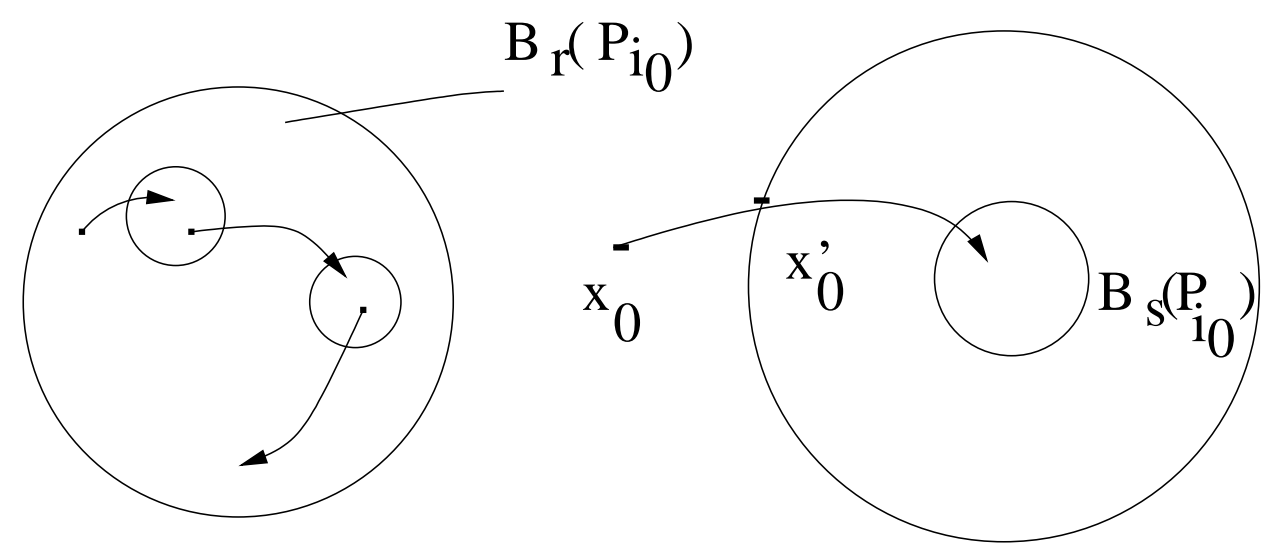

(1)

(5)

Here $C_{2}$ depends only on $s_{0}, \alpha$ and $r$.

For $N$,

$$
N \leq 2 k M_{\alpha}(r)
$$

Combining the above estimates, we have

$$
\left|\int_{\bar{\gamma}} \alpha\right| \leq k C \leq n C
$$

where $C$ depends on $r, s_{0}, \alpha$ and $\max |\dot{v}(t)|$.

Now the proof of the proposition is changed to the problem to reduce each $\left(\mathcal{U}(s), T\left(s_{0}\right)\right)$-chain to an $\left(r, s_{0}\right)$-chain, while keeping the integral of $\alpha$ invariant.

Let $\tilde{\gamma}=\left\{x_{0}, x_{1}, \cdots, x_{k} \mid t_{0}, \cdots, t_{k-1}\right\}$ be a $\left(\mathcal{U}(s), T\left(s_{0}\right)\right)$-chain. Consider the following cases.

(1) If the chain $\tilde{\gamma}$ is contained in a ball $\bar{B}_{r}\left(p_{i_{0}}\right)$ for $0 \leq i_{0} \leq n$, then

$$
\begin{aligned}
& \left|\int_{\tilde{\gamma}} \alpha\right|=\left|\beta_{p_{i_{0}}}\left(x_{k}\right)-\beta_{p_{i_{0}}}\left(x_{0}\right)\right| \\
& \leq \operatorname{osc}_{x \in B_{r}\left(p_{i_{0}}\right)} \beta_{p_{i_{o}}}(x) \leq 2 M_{\alpha}(r)
\end{aligned}
$$

(2) There is $k_{0}, 1 \leq k_{0} \leq k$ such that the subchain $\tilde{\gamma}_{0 k_{0}}=\left\{x_{0}, x_{1}, \cdots, x_{k_{0}} \mid t_{0}, \cdots\right.$, $\left.t_{k_{0}-1}\right\}$ is contained in $B_{r}\left(p_{i_{0}}\right)$ but $\left[x_{k_{0}}, x_{k_{0}} \cdot t_{k_{0}}\right]$ does not intersect $\cup_{j \neq i_{0}}^{n} \bar{B}_{r}\left(p_{j}\right)$. Hence there is a first intersection point $\bar{x}_{0}$ of $\left[x_{k_{0}}, x_{k_{0}} \cdot t_{k_{0}}\right]$ with $\partial B_{r}\left(p_{i_{0}}\right)$. Let $\bar{t}_{0}$ satisfy $\bar{x}_{0} \cdot \bar{t}_{0}=x_{k_{0}} \cdot t_{k_{0}}$ and let $\bar{y}_{0}=x_{k_{0}+1}$. Denote the ball containing the point pair $\left(\bar{x}_{0} \cdot \bar{t}_{0}, \bar{y}_{0}\right)$ by $B_{s}\left(m_{0}\right)$. Since $t_{k_{0}} \geq T\left(s_{0}\right)$ and $\tilde{\gamma}_{0 k_{0}} \subset B_{r}\left(p_{i_{0}}\right)$, this implies that $x_{k_{0}} \in B_{s_{0}}\left(p_{i_{0}}\right)$, hence the trajectory $\left[\bar{x}_{0}, \bar{x}_{0} \cdot \bar{t}_{0}\right]$ does not intersect with $B_{s_{0}}\left(p_{i_{0}}\right)$. Otherwise, $\left[x_{k_{0}}, x_{k_{0}} \cdot t_{k_{0}}\right]$ starts from $B_{s_{0}}\left(p_{i_{0}}\right)$, intersects $\partial B_{r}\left(p_{i_{0}}\right)$, then goes back to $B_{s_{0}}\left(p_{i_{0}}\right)$. This conclusion contradicts (3.3) when we check the change of the Lyapounov function $g(x)$ along $\left[x_{k_{0}}, x_{k_{0}} \cdot t_{k_{0}}\right]$. Therefore $\left[\bar{x}_{0}, \bar{x}_{0} \cdot \bar{t}_{0}\right]$ 


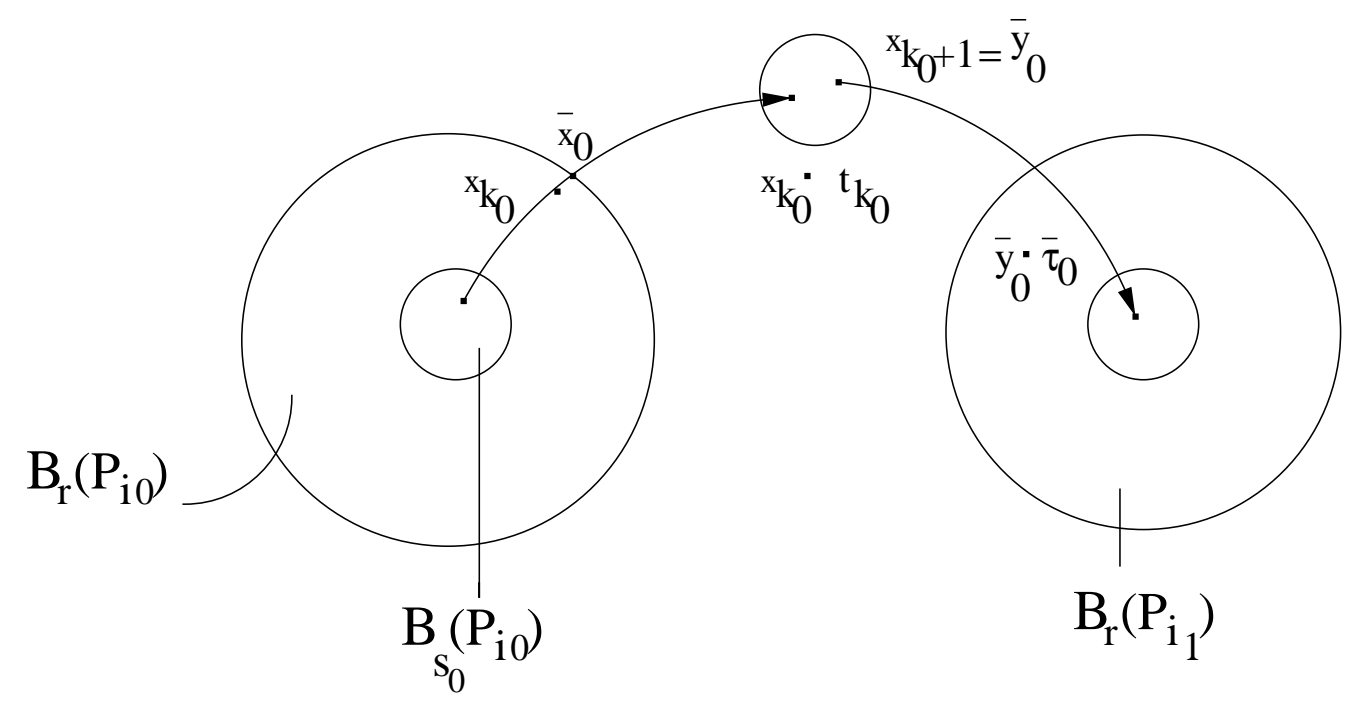

(2)

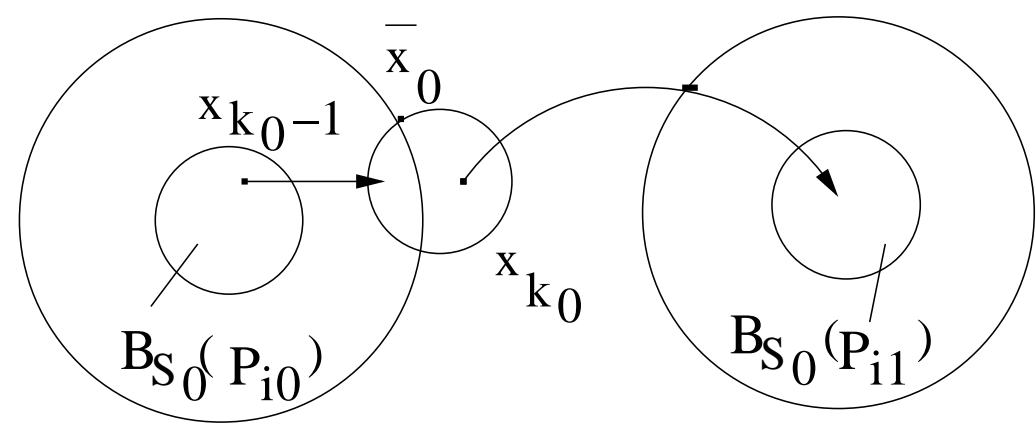

(3)

is part of a trajectory in $\Gamma_{1}\left(s_{0}\right)$, and we have $\bar{t}_{0} \leq T\left(s_{0}\right)$. Since $\bar{x}_{0} \cdot \overline{t_{0}}=x_{k_{0}} \cdot t_{k_{0}}$ is not in $\bar{B}_{r}\left(p_{i_{0}}\right)$, by the choice of $s$, we know that $\bar{y}_{0}:=x_{k_{0}+1} \notin B_{s_{0}}\left(p_{i_{0}}\right)$. Hence $\bar{y}_{0} \cdot t_{k_{0}+1}$ meets firstly $B_{s_{0}}\left(p_{l_{1}}\right)$ for some fixed point $p_{l_{1}}$. Let $\bar{y}_{0} \cdot \bar{\tau}_{0}$ be the last intersection point of $\left[\bar{y}_{0}, \bar{y}_{0} \cdot t_{k_{0}+1}\right]$ with $\partial B_{r}\left(p_{i_{1}}\right)$. In the same way, we can prove that $\bar{\tau}_{0} \leq T\left(s_{0}\right)$. Joining $x_{0}$ and $\bar{x}_{0}$ by a curve $l_{x_{0} \bar{x}_{0}}$, then the subchain $\tilde{\gamma}_{0 k_{0}}$ is reduced to a curve $l_{x_{0} \bar{x}_{0}}$ combined with a chain $\left\{\bar{x}_{0}, \bar{y}_{0} \mid \bar{t}_{0}, \bar{\tau}_{0}\right\}$ and it is clear that the reduction keeps the integral of $\alpha$ invariant.

(3) There is a $k_{0}, 2 \leq k_{0} \leq k$ such that the subchain $\tilde{\gamma}_{0, k_{0}-1}=\left\{x_{0}, x_{1}, \cdots, x_{k_{0}-1} \mid t_{0}\right.$, 


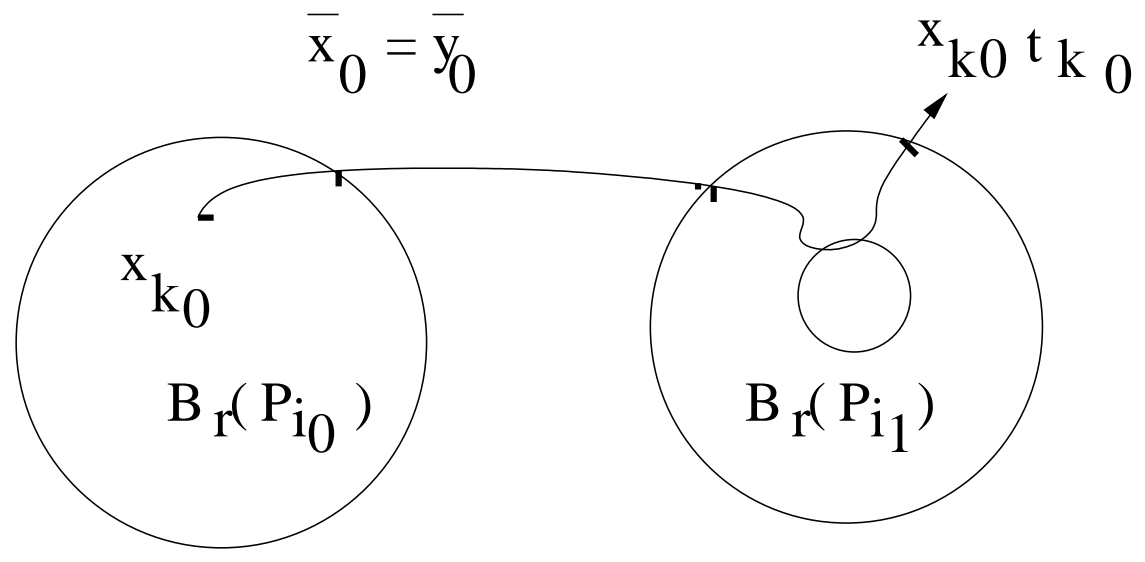

(4)

$\left.t_{1}, \cdots, t_{k_{0}-2}\right\}$ and the trajectory $\left[x_{k_{0}-1}, x_{k_{0}-1} \cdot t_{k_{0}-1}\right]$ is contained in $B_{r}\left(p_{i_{0}}\right)$ but $x_{k_{0}} \notin B_{r}\left(p_{i_{0}}\right)$. Then we take a point $\bar{x}_{0} \in \partial B_{r}\left(p_{i_{0}}\right) \cap B_{s}\left(m_{0}\right)$, where $B_{s}\left(m_{0}\right)$ is the ball containing $\left(x_{k_{0}-1} \cdot t_{k_{0}-1}, x_{k_{0}}\right)$. Since $t_{k_{0}} \geq T\left(s_{0}\right)$, the trajectory $\left[x_{k_{0}}, x_{k_{0}} \cdot t_{k_{0}}\right]$ will meet $B_{s_{0}}\left(p_{i_{1}}\right)$. We denote $x_{k_{0}}$ by $\bar{y}_{0}$ and represent the last intersection point of $\left[x_{k_{0}}, x_{k_{0}} \cdot t_{k_{0}}\right]$ with $\partial B_{r}\left(p_{i_{1}}\right)$ by $\bar{y}_{0} \bar{\tau}_{0}$ for some $\bar{\tau}_{0}>0$. Due to the same argument as in (2), we know that $\bar{\tau}_{0} \leq T\left(s_{0}\right)$. So $\tilde{\gamma}_{0 k_{0}}$ is reduced to the combination of a curve $l_{x_{0} \bar{x}_{0}}$ and a chain $\left\{\bar{x}_{0}, \bar{y}_{0} \mid 0, \bar{\tau}_{0}\right\}$. It is clear that such inductions also keep the integral of $\alpha$ invariant. Note that $x_{k_{0}-1}$ must be in $B_{s_{0}}\left(p_{i_{0}}\right)$.

(4) There is a subchain $\tilde{\gamma}_{0 k_{0}}=\left\{x_{0}, x_{1}, \cdots, x_{k_{0}} \mid t_{0}, \cdots, t_{k_{0}-1}\right\}$ contained in $B_{r}\left(p_{i_{0}}\right)$ and the trajectory $\left[x_{k_{0}}, x_{k_{0}} \cdot t_{k_{0}}\right]$ goes through $B_{s_{0}}\left(p_{i_{1}}\right)$. Let $\bar{x}_{0}=\bar{y}_{0}$ be the first intersection point of $\left[x_{k_{0}}, x_{k_{0}} \cdot t_{k_{0}}\right]$ with $\partial B_{r}\left(p_{i_{0}}\right)$ and let $\bar{y}_{0} \cdot \bar{\tau}_{0}$ be the last intersection point of $\left[x_{k_{0}}, x_{k_{0}} \cdot t_{k_{0}}\right]$ with $\partial B_{r}\left(p_{i_{1}}\right)$ before hitting $B_{s}\left(p_{i_{1}}\right)$. Also we can prove that $\bar{\tau}_{0} \leq T\left(s_{0}\right)$ as in (2) and (3). Then the chain $\tilde{\gamma}_{0 k_{0}}$ followed by $\left[x_{k_{0}}, \bar{y}_{0} \cdot \bar{\tau}_{0}\right]$ is reduced to the combination of $l_{x_{0} \bar{x}_{0}}$ with the chain $\left\{\bar{x}_{0}, \bar{y}_{0} \mid 0, \bar{\tau}_{0}\right\}$, while the integral of $\alpha$ is invariant under change.

(5) If $x_{0} \notin \cup_{j=1}^{n} B_{r}\left(p_{j}\right)$, then $\left[x_{0}, x_{0} \cdot t_{0}\right]$ will firstly meet some $B_{s}\left(p_{i_{0}}\right)$. Let $x_{0}^{\prime}=x_{0} \cdot t_{0}^{\prime}$ be the last intersection point of $\left[x_{0}, x_{0} \cdot t_{0}\right]$ with $\partial B_{r}\left(p_{i_{0}}\right)$. In the same way, we can prove $t_{0}^{\prime} \leq T\left(s_{0}\right)$.

Now using the above steps (2)-(5) repeatedly, any $\left(\mathcal{U}(s), T\left(s_{0}\right)\right)$ - chain $\tilde{\gamma}=$ $\left\{x_{0}, x_{1}, \cdots, x_{k} \mid t_{0}, \cdots, t_{k-1}\right\}$ can be reduced to the combination of a curve $l_{x_{0} \bar{x}_{0}}$ ( or $\left[x_{0}, x_{0} \cdot t_{0}^{\prime}\right]$ in case $\left.(5)\right)$ with an $\left(r, s_{0}\right)$-chain $\bar{\gamma}=\left\{\bar{x}_{0}, \bar{y}_{0}, \cdots, \bar{x}_{l} \mid \bar{t}_{0}, \bar{\tau}_{0}, \cdots, \bar{\tau}_{l-1}\right\}$ if we can prove that (4) in the definition of a $\left(r, s_{0}\right)$-chain holds.

If (4) is not true, then there exists an $\left(r, s_{0}\right)$-cycle $\bar{\gamma}_{c}=\left\{\bar{x}_{i_{0}}, \bar{y}_{i_{0}}, \cdots, \bar{y}_{i_{l}}, \bar{x}_{i_{0}} \mid \bar{t}_{i_{0}}\right.$, $\left.\bar{\tau}_{i_{0}}, \cdots, \bar{\tau}_{i_{l}}\right\}$ where the point pair $\left(\bar{y}_{i_{l}} \cdot \bar{\tau}_{i_{l}}, \bar{x}_{i_{0}}\right) \in \partial B_{r}\left(p_{i_{0}}\right)$. 
Consider the change of the function $g(x)$ along $\tilde{\gamma}_{c}$. On one hand, since the two ends of $\tilde{\gamma}_{c}$ are $x_{i_{0}}$ and $\bar{y}_{i_{l}} \cdot \tau_{i_{l}}$, by (3.3)

$$
\left|\int_{\tilde{\gamma}_{c}} \delta g\right| \leq \operatorname{osc}_{x \in B_{s_{0}}\left(p_{i_{0}}\right)} g(x)<\frac{1}{3} \delta_{0}(r)<\frac{1}{12} \epsilon_{0},
$$

where $\epsilon_{0}$ is from the requirement to the Lyapunov function $g$. On the other hand, we have

$$
\int_{\tilde{\gamma}_{c}} \delta g \geq k \epsilon_{0}-k \max _{j} \operatorname{osc}_{y \in B_{r}\left(p_{j}\right)} g(y)-k \max _{j} \operatorname{osc}_{y \in B_{s}\left(m_{j}\right)} g(y)>\epsilon_{0} / 2
$$

This is absurd. The contradiction shows that the chain $\bar{\gamma}$ we get from a $\left(\mathcal{U}(s), T\left(s_{0}\right)\right)$ chain $\tilde{\gamma}$ is indeed an $\left(r, s_{0}\right)$-chain. Let $\bar{\gamma}=\left\{\bar{x}_{0}, \bar{y}_{0}, \cdots, \bar{x}_{l} \mid \bar{t}_{0}, \bar{\tau}_{0}, \cdots, \bar{\tau}_{l-1}\right\}$. Therefore applying the estimate (3.6) and the fact that the reduction from $\tilde{\gamma}$ to $\bar{\gamma}$ keeps the integral of $\alpha$, we have

$$
\left|\int_{\tilde{\gamma}} \alpha\right|=\left|\int_{\bar{\gamma}} \alpha\right| \leq C
$$

where $C$ depends on $r, s_{0}, \alpha$ and $\max |\dot{v}(t)|$. Theorem 3.2 now is proved.

Theorem 3.3 Let $v$ be an $\alpha$-flow with a nontrivial cocycle $\alpha$ on a compact metric space $(X, d)$. Assume that the sequence $\left\{s_{i}\right\} \rightarrow 0$ as $i \rightarrow \infty$. If for any $M>0$ and $T>0$, there is a $i_{0} \in \mathbb{N}$ such that there exists a $\left(\mathcal{U}\left(s_{i}\right), T\right)$-chain $\tilde{\gamma}_{i}$ for each $i \geq i_{0}$ satisfying

$$
\left|\int_{\tilde{\gamma}_{i}} \alpha\right| \geq M
$$

then $v$ is not a gradient-like flow. Furthermore, if it is Known that for any $M>0$, there is a trajectory $\tilde{\gamma}$ satisfying (3.7) or there is an oriented cycle $\tilde{\gamma}_{0}$ consisting of some orbits joining fixed points where the direction is determined by the forward direction of the flow $v$ such that

$$
\left|\int_{\tilde{\gamma}} \alpha\right| \geq 1
$$

then $v$ is not a gradient-like flow.

Proof. The first conclusion is a direct corollary of Theorem 3.2 and the third one is obvious. We only consider the second case. It is easy to see that if the time intervals of the trajectories have an upper bound, then for any cocycles on $X$ the absolute value of the integral, $\left|\int_{\tilde{\gamma}} \alpha\right|$ has a uniform bound. Therefore the trajectory $\tilde{\gamma}$ with respect to the arbitrary large $M$ has arbitrary large time interval and it is the chain needed for the hypothesis in the first conclusion.

Theorem 3.4 Let $v$ be an $\alpha$-flow with a nontrivial cocycle $\alpha$ on a compact metric space $(X, d)$. Assume that the sequence $\left\{s_{i}\right\} \rightarrow 0$ as $i \rightarrow \infty$. If $v$ is not 
a gradient-like flow, then for any $M>0$ and $T>0$, there is a $i_{0} \in \mathbb{N}$ such that there exists a $\left(\mathcal{U}\left(s_{i}\right), T\right)$-chain $\tilde{\gamma}_{i}$ for each $i \geq i_{0}$ satisfying

$$
\left|\int_{\tilde{\gamma}_{i}} \alpha\right| \geq M
$$

Proof Firstly by the same argument as in the proof of Theorem 3.2, we can obtain a upper bound $T\left(\frac{r}{4}\right)$ for the time interval of any trajectory $\gamma \in \Gamma_{1}\left(\frac{r}{4}\right)$. If $v$ is not a gradient-like flow, then there is a non fixed point $x_{0}$ in the chain recurrent set of $v$. Therefore for any $s_{i}$ and $T>2 T\left(\frac{r}{4}\right)$ there is a $\left(\mathcal{U}\left(s_{i}\right), T\right)$-chain $\tilde{\gamma}_{i}=\left\{x_{0}, \cdots, x_{k}=x_{0} \mid t_{0}, \cdots, t_{k-1}\right\}$.

Now we consider two possibilities:

(1). If there exists $x_{i}$ such that for any $t_{0}>0 x_{i} \cdot t \notin \cup_{i=1}^{n} p_{i}\left(\frac{r}{4}\right)$ for some $t>t_{0}$, where $p_{i}(r):=A_{i}\left(V_{i}, r\right)$ is the flow neighborhood of $p_{i}$.

There are two cases. The first case is that after time $\tau, x_{i} \cdot t$ becomes a flow line in $\Gamma_{2}\left(\frac{r}{4}\right) \cup \Gamma_{3}\left(\frac{r}{4}\right)$. Then

$$
\int_{\left[x_{i}, x_{i} \cdot t\right]} \alpha \geq\left[\frac{t-\tau}{T_{0}}\right] \rho-C,
$$

where $\tau, C>0$ are constants. Hence we can take the trajectory $\left[x_{i}, x_{i} \cdot t\right]$ as the required $\left(\mathcal{U}\left(s_{i}\right), T\right)$-chain for $T$ arbitrary large. The second case is that $x_{i} \cdot t$ can meet infinitely many flow neighborhoods $\left\{p_{i_{0}}(r), \cdots, p_{i_{j}}(r), \cdots\right\}$, then

$$
\int_{\left[x_{i}, x_{i} \cdot t\right]} \alpha \geq \sum_{k=0}^{l(t)-1} \int_{\left[x_{i}, x_{i} \cdot t\right]_{k}} \alpha-\sum_{k=1}^{l(t)-1} \operatorname{osc}_{x \in p_{l_{k}}(r)} \beta_{p_{l_{k}}}(x),
$$

where $l(t)$ is an integer representing the number of flow neighborhoods that $\left[x_{i}, x_{i}\right.$. $t]$ has met and $\left[x_{i}, x_{i} \cdot t\right]_{k} \in \Gamma_{1}(r)$ is a trajectory of $\left[x_{i}, x_{i} \cdot t\right]$ between $p_{i_{k}}(r)$ and $p_{i_{k+1}}(r)$. We have

$$
\int_{\left[x_{i}, x_{i} \cdot t\right]} \alpha \geq l(t)(1-\lambda) \rho .
$$

If $t$ is large enough, this integral is larger than any given number. Hence we can take $\left[x_{i}, x_{i} \cdot t\right]$ as the required chain.

(2). For any $i$, there exists a $t_{0}>0$ such that $x_{i} \cdot t \in \cup_{i=1}^{n} p_{i}\left(\frac{r}{4}\right)$ for $t>t_{0}$.

Consider the homeomorphism $\varphi_{T\left(\frac{r}{4}\right)}: X \rightarrow X$ which maps $x$ to $x \cdot T\left(\frac{r}{4}\right)$. Let $B_{s}$ be any balls in $\mathcal{U}(s)$, then by compactness the upper bound of the diameter of $\varphi_{t}\left(B_{s}\right), \forall t \in\left[0, T\left(\frac{r}{4}\right)\right]$, will be less than a uniform constant $C_{s}<\frac{r}{4}$ if $s$ is small enough.

Since $\left[x_{0}, x_{0} \cdot t_{0}\right]$ will meet the first flow neighborhood $p_{i_{0}}\left(\frac{r}{4}\right)$, we can replace $x_{0}$ by $x_{0} \cdot t_{0}$, since $x_{0} \cdot t_{0}$ is also a point in the chain recurrent set of the flow 
$v$. So we assume that $x_{0} \in p_{i_{0}}\left(\frac{r}{4}\right)$. Now we want to modify the $\left(\mathcal{U}\left(s_{i}\right), T\right)$ chain $\tilde{\gamma}_{i}=\left\{x_{0}, \cdots, x_{k}=x_{0} \mid t_{0}, \cdots, t_{k-1}\right\}$ to another $\left(\mathcal{U}(r), T\left(\frac{r}{4}\right)\right)$-chain $\gamma_{i}^{\prime}=$ $\left\{x_{0}^{\prime}, \cdots, x_{k}^{\prime}=x_{0}^{\prime} \mid t_{0}^{\prime}, \cdots, t_{k-1}^{\prime}\right\}$ satisfying that the pair $\left(x_{j}^{\prime} \cdot t_{j}^{\prime}, x_{j+1}^{\prime}\right) \in p_{i_{j}+1}(r)$ for $j=0,1, \cdots, k-1$. If $B_{s_{i}}\left(m_{1}\right)$ is contained in some $p_{i_{1}}(r)$, then we are done. Assume that $B_{s_{i}}\left(m_{1}\right)$ is not contained in any $p_{i}(r)$. By the choice of $T\left(\frac{r}{4}\right)$, the flow line $\left[x_{1}, x_{1} \cdot T\left(\frac{r}{4}\right)\right]$ will intersect some flow neighborhood $p_{i_{1}}\left(\frac{r}{4}\right)$ at some point $x_{1}^{\prime}=x_{1} \cdot \tau_{1} \in B_{\frac{r}{4}}\left(p_{i_{1}}\right)$. If $s_{i}$ is small enough, then $\left(x_{0} \cdot\left(t_{0}+\tau_{1}\right) \in B_{\frac{r}{2}}\left(p_{i_{1}}\right)\right)$, hence in $p_{i_{1}}(r)$. Let $x_{0}^{\prime}=x_{0}, x_{1}^{\prime}=x_{1} \cdot \tau_{1}, t_{0}^{\prime}=t_{0}+\tau_{1}$ and $t_{1}^{\prime}=t_{1}-\tau_{1}$. Hence we get the modified chain $\left\{x_{0}^{\prime}, x_{1}^{\prime}, x_{2}, \cdot, x_{k} \mid t_{0}^{\prime}, t_{1}^{\prime}, t_{2}, \cdots\right\}$. Now $x_{1}^{\prime} \in p_{i_{1}}\left(\frac{r}{4}\right)$, we can continue this operation until we get the modified chain $\gamma_{i}^{\prime}$. So if $s_{i}$ is small, we have

$$
\int_{\tilde{\gamma}_{i}} \alpha=\int_{\gamma_{i}^{\prime}} \alpha \geq \sum_{j=0}^{k-1}\left(\int_{\left[x_{j}^{\prime}, x_{j}^{\prime} \cdot t_{j}^{\prime}\right]}-\operatorname{osc}_{x \in p_{i_{j}}(r)} \beta_{p_{i_{j}}}(x)\right) \geq k(1-\lambda) \rho>0 .
$$

Here the first equality is because the integration of $a$ only depends on the relative cohomology class of the path. So if $n$ is large enough, the integral of $\alpha$ along $n \tilde{\gamma}_{i}$ will be larger than any given number. Hence (3.9) holds.

In view of Theorem 3.2-3.4, the following corollary is obvious.

Corollary 3.5 Let $v$ be an $\alpha$-flow on the compact metric space $(X, d)$. If for any chain $\tilde{\gamma}$,

$$
\left|\int_{\tilde{\gamma}} \alpha\right| \leq M
$$

for some $M>0$, then $v$ is a gradient-like flow.

Example 3.2 Let $v$ be a flow on $S^{1}$ which has three fixed points at $\theta=0, \frac{2 \pi}{3}, \frac{4 \pi}{3}$, and the forward direction of the flow is the anticlockwise direction. It is easy to see that $v$ carries a nontrivial cocycle and is a non gradient-like flow.

\section{An algebraic theorem relative to $\pi$-Morse decomposition}

In this section, we let $X$ be an $m$-dimensional compact polyhedron with metric. Let $v$ be a $\alpha$-flow on $X$, where $\alpha$ is a nontrivial cocycle with higher rank.

By Theorem 3.4.4 in [FJ2], the flow $v$ has a $\pi$-Morse decomposition, where $\pi$ is the deck transformation group on the covering space $\bar{X}$ determined by the cocycle $\alpha$. Assume that $\pi$ is spanned by $G_{+}:=\left\{l_{1}, \cdots, l_{s}\right\}$, where $s$ is the rank of $\alpha$. By the proof of Theorem 3.4.3 in [FJ2], we can get $s$ pair cross sections $N_{i}^{ \pm}$ that satisfy the relations:

$$
l_{i} \cdot N_{i}^{-}=N_{i}^{+}, i=1, \cdots, s .
$$

The union $\cup_{i=1}^{s}\left(N_{i}^{+} \cup N_{i}^{-}\right)$forms the boundary of a fundamental domain $X_{0}$. Since $X$ is a compact polyhedron, we can take an $m$-1-dimensional polyhedron 


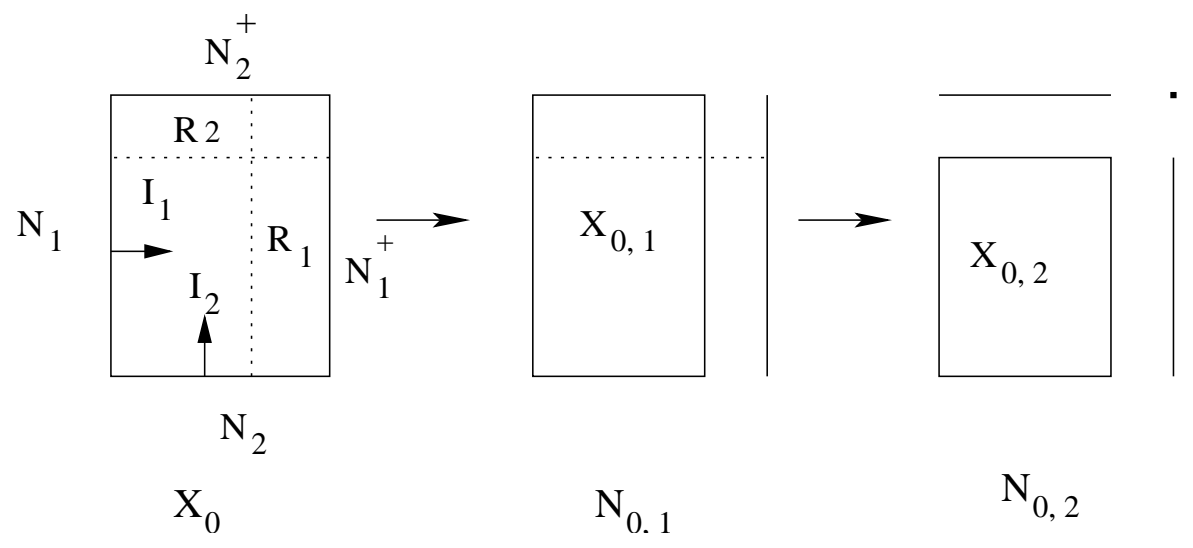

Figure 1. Cell decomposition

approximating the cross sections $N_{i}^{-}$for $i=1, \cdots, s$ such that the intersections $N_{\alpha}^{-}=N_{i_{1}}^{-} \cap N_{i_{2}}^{-} \cap \cdots N_{i_{r}}^{-}, \alpha=\left\{i_{1} \cdots, i_{r}\right\}$ are polyhedra with codimension $r$. So without loss of generality, we can assume the cross sections $N_{i}^{ \pm}$and their intersections are polyhedra. For convenience, we denote $N_{i}^{-}=N_{i}$.

Cell decomposition of the fundamental domain We can retract $N_{i}^{+}$a small distance $\epsilon$ into the inner part of the fundamental domain to get a homeomorphic codimension 1 polyhedron (see Figure 1 . for $s=2$ case). We denote the obtained polyhedron as $R_{i}$. Let $i_{j,+}: R_{j} \rightarrow X_{0}, j=1, \cdots, s$ be the inclusion and $i_{j,-}: R_{j} \rightarrow N_{j}$ be the homeomorphism.

Let $I^{s}=(0,1)$ be the 1-dimensional cell in the $s-t h$ direction. Firstly we delete $R_{1} \times \epsilon I^{1}$ and get a polyhedron $N_{0,1}$. Denote $X_{0,1}=N_{0,1} / N_{1}^{+}$. We call $G_{1}=\left(X_{0,1}, N_{0,1}, R_{1}, i_{1, \pm}\right)$ to be the first gluing data. Assume after $r$-th excision, one has the gluing data $G_{r}=\left(X_{0, r}, N_{0, r}, R_{1}, \cdots, R_{r}, i_{1, \pm}, \cdots, i_{r, \pm}\right)$. Now deleting $R_{r+1} \times \epsilon I^{r+1}$ from $N_{0, r}$, we get the polyhedron $N_{0, r+1}$. Define $X_{0, r+1}=N_{0, r+1}-\cup_{i=1}^{r+1} N_{i}^{+}$. Hence after $r+1$-th excision, one has the gluing data $G_{r+1}$. Continue this process until we get the $s$-th gluing data $G_{s}=$ $\left(X_{0, s}, N_{0, s}, R_{1}, \cdots, R_{s}, i_{1, \pm}, \cdots, i_{s, \pm}\right) . G_{i}$ is called the $i$-th gluing data because starting from these data, one can reconstruct and give a compatible cell decomposition to the fundamental domain $X_{0}$ through $i$ times inverse attaching operations.

Now we want to construct a compatible regular cell decomposition of $X_{0}$. Firstly we chose an arbitrary cell decomposition of $\cup_{i=1}^{s}\left(R_{i}^{\circ} \cap X_{0, s}\right)$, where $R_{i}^{\circ}$ is the interior part of $R_{i}$. Then the homeomorphisms $i_{j,-}, j=1, \cdots, s$ provide a 
cell decomposition of the other half part of the boundary of $X_{0, s}$. In the interior of $X_{0, s}$, we choose an arbitrary compatible regular cell decomposition. Then we get a cell decomposition of $X_{0, s}$. Now the action of the group provides a cell decomposition of $N_{0, s}$. At this moment, we can use our gluing data to add the cylindrical cells to $N_{0, s}$. We add firstly the cell $e \times \epsilon I^{s}$ for $e \in R_{s} \cap N_{0, s}$ to $N_{0, s}$ by the inclusion map $i_{s,+}$ and by identifying the end $e \times \epsilon\{1\}$ to $l_{s} \cdot i_{s,-}(e)$. So we obtain a cell decomposition of $N_{0, s-1}$ with the gluing data $G_{s-1}$. Continuing this process, we can obtain a compatible regular cell decomposition (i.e., each closed sets appeared here are all cell subcomplexes) of $X_{0}$.

Since $N_{0, s}$ is a cell complex, we can choose arbitrary orientation of a cell $e \in C_{q}\left(N_{0, s}\right)$. Hence fixing the incidence coefficients appearing in the boundary operator of the cell chain complex. For the orientation of the cylindrical cells $e \times I^{i_{1}} \times \cdots I^{i_{k}}$, we choose the natural orientation, i.e., the order $i_{1}<i_{2} \cdots<i_{k}$ defines the positive orientation. The orientation of the product cell $e \times I^{i_{1}} \times \cdots I^{i_{k}}$ is uniquely determined by the orientation of the factor cells. So the incidence coefficients of the boundary operator of $C_{*}\left(X_{0}\right)$ are uniquely determined.

Deformation of cell chain complexes Let $\alpha \in C_{q}\left(X_{0}\right)$, then there is a unique decomposition

$$
\alpha=(-1)^{q-1}\left(i_{1,+}\right)_{*}\left(\alpha_{1}\right) \times \epsilon I^{1}+\alpha_{1}^{\prime} .
$$

Define $\beta_{1}(\alpha)=\alpha_{1}, \beta_{1}^{\prime}(\alpha)=\alpha_{1}^{\prime}$ and $\theta_{1}\left(\alpha_{1}\right)=(-1)^{q-1}\left(i_{1,+}\right)_{*}\left(\alpha_{1}\right) \times \epsilon I^{1}$. We have the relation

$$
\left(\theta_{1}, 1\right)\left(\begin{array}{c}
\beta_{1} \\
\beta_{1}^{\prime}
\end{array}\right)=I d
$$

Similarly, for $\alpha \in C_{q}\left(N_{0, k-1}\right)$ we have the decomposition

$$
\alpha=(-1)^{q-1}\left(i_{k,+}\right)_{*}\left(\alpha_{k}\right) \times \epsilon I^{k}+\alpha_{k}^{\prime} .
$$

Let $\beta_{k}(\alpha):=\alpha_{k} \in C_{q-1}\left(R_{k}-\cup_{i=1}^{k-1} R_{i} \times I^{i}\right), \beta_{k}^{\prime}(\alpha):=\alpha_{k}^{\prime} \in C_{q}\left(N_{0, k}\right)$ and $\theta_{k}\left(\alpha_{k}\right):=(-1)^{q-1}\left(i_{k,+}\right)_{*}\left(\alpha_{k}\right) \times \epsilon I^{k}$. We have the equalities

$$
\left(\theta_{k}, 1\right)\left(\begin{array}{c}
\beta_{k} \\
\beta_{k}^{\prime}
\end{array}\right)=I d, i=2, \cdots, s
$$

Lemma 4.1 $\theta_{k}, \beta_{k}, \beta_{k}^{\prime}$ are maps satisfying the following equalities:

(1) $d_{N_{0, k-1}} \circ \theta_{k}+\theta_{k} \circ d_{R_{k}}=l_{k} \cdot\left(i_{k,-}\right)_{*}-\left(i_{k,+}\right)_{*}$

(2) $\beta_{k} \circ d_{N_{0, k-1}}+d_{R_{k}} \circ \beta_{k}=0$

(3) $\beta_{k}^{\prime} \circ d_{N_{0, k-1}}-d_{N_{0, k}} \circ \beta_{k}^{\prime}=\left(l_{k} \cdot\left(i_{k,-}\right)_{*}-\left(i_{k,+}\right)_{*}\right) \circ \beta_{k}$

The above relations can also be written in matrix form: 
$(4)$

(5)

$$
d_{N_{0, k-1}} \circ\left(\theta_{k}, 1\right)=\left(\theta_{k}, 1\right)\left(\begin{array}{cc}
-d_{R_{k}} & 0 \\
f_{k} & d_{N_{0, k}}
\end{array}\right)
$$

$$
\left(\begin{array}{c}
\beta_{k} \\
\beta_{k}^{\prime}
\end{array}\right) \circ d_{N_{0, k-1}}=\left(\begin{array}{cc}
-d_{R_{k}} & 0 \\
f_{k} & d_{N_{0, k}}
\end{array}\right)\left(\begin{array}{c}
\beta_{k} \\
\beta_{k}^{\prime}
\end{array}\right)
$$

Here $f_{k}:=l_{k} \cdot\left(i_{k,-}\right)_{*}-\left(i_{k,+}\right)_{*}$.

Proof

(1) Let $\alpha \in C_{q-1}\left(R_{k}-\cup_{i=1}^{k-1} R_{i} \times I^{i}\right)$, then

$$
\begin{aligned}
& d_{N_{0, k-1}} \circ \theta_{k}(\alpha)=d_{N_{0, k-1}}\left((-1)^{q-1}\left(i_{k,+}\right)_{*}(\alpha) \times \epsilon I^{k}\right) \\
& =(-1)^{q-1}\left(i_{k,+}\right)_{*}\left(d_{R_{k}} \alpha\right) \times \epsilon I^{k}+\left(l_{k} \cdot\left(i_{k,-}\right)_{*}-\left(i_{k,+}\right)_{*}\right)(\alpha) \\
& =-\theta_{k} \circ d_{R_{k}} \alpha+\left(l_{k} \cdot\left(i_{k,-}\right)_{*}-\left(i_{k,+}\right)_{*}\right)(\alpha)
\end{aligned}
$$

(2) Assume $\alpha \in C_{q-1}\left(N_{0, k-1}\right)$ has the form

$$
\alpha=(-)^{q-2}\left(i_{k,+}\right)_{*}\left(e_{1}\right) \times \epsilon I^{k}+e_{2},
$$

where $e_{1} \in C_{q-2}\left(R_{k}-\cup_{i=1}^{k-1} R_{i} \times \epsilon I^{i}\right)$ and $e_{2} \in C_{q-1}\left(N_{0, k}\right)$. Then

$$
d_{N_{0, k-1}} \alpha=(-)^{q-2}\left(i_{k,+}\right)_{*}\left(d_{R_{k}} e_{1}\right) \times \epsilon I^{k}+\left(l_{k} \cdot\left(i_{k,-}\right)_{*}-\left(i_{k,+}\right)_{*}\right)\left(e_{1}\right)+d_{N_{0, k}} e_{2},
$$

hence

$$
\beta_{k} \circ d_{N_{0, k-1}} \alpha=-d_{R_{k}} \circ \beta_{k}(\alpha)
$$

and

$$
\beta_{k}^{\prime} \circ d_{N_{0, k-1}} \alpha=\left(l_{k} \cdot\left(i_{k,-}\right)_{*}-\left(i_{k,+}\right)_{*}\right) \circ \beta_{k}(\alpha)+d_{N_{0, k}} \circ \beta_{k}^{\prime}(\alpha)
$$

Lemma 4.2 Let $f_{k}:=l_{k} \cdot\left(i_{k,-}\right)_{*}-\left(i_{k,+}\right)_{*}, k=1, \cdots, s$ be the chain map from $C_{*-1}\left(R_{k}-\cup_{i=1}^{k-1} R_{i} \times I^{i}\right)$ to $C_{*-1}\left(N_{0, k}\right)$. Then $\left(\theta_{k}, 1\right)$ is a chain isomorphism from the cone con $\left(f_{k}\right):=\left(C_{*-1}\left(R_{k}-\cup_{i=1}^{k-1} R_{i} \times I^{i}\right) \oplus C_{*-1}\left(N_{0, k}\right), d_{c}^{k}\right)$ to $\left(C_{*}\left(N_{0, k-1}\right), d_{N_{0, k-1}}\right)$, where the differential of the algebraic cone is

$$
d_{c}^{k}:=\left(\begin{array}{cc}
-d_{R_{k}} & 0 \\
f_{k} & d_{N_{0, k}}
\end{array}\right)
$$

Proof. By equalities (4) and (5) of Lemma 4.1, $\left(\theta_{k}, 1\right)$ and $\left(\begin{array}{c}\beta_{k} \\ \beta_{k}^{\prime}\end{array}\right)$ are chain maps with respect to corresponding cellular chain complexes. Furthermore, we have

$$
\left(\theta_{k}, 1\right)\left(\begin{array}{c}
\beta_{k} \\
\beta_{k}^{\prime}
\end{array}\right)=I d
$$


and

$$
\left(\begin{array}{c}
\beta_{k} \\
\beta_{k}^{\prime}
\end{array}\right)\left(\theta_{k}, 1\right)=\left(\begin{array}{ll}
1 & 0 \\
0 & 1
\end{array}\right) .
$$

Therefore $\left(\theta_{k}, 1\right)$ is a chain isomorphism.

\section{Lemma 4.3 Let}

$$
\begin{gathered}
D_{q}^{k}=C_{q-1}\left(R_{1}\right) \oplus C_{q-1}\left(R_{2}-\left(i_{1,+}\right)\left(R_{1}\right) \times \epsilon I^{1}\right) \oplus \cdots \\
\oplus C_{q-1}\left(R_{k}-\cup_{j=1}^{k-1}\left(i_{j,+}\right)\left(R_{j}\right) \times \epsilon I^{j}\right) \otimes C_{q}\left(N_{0, k}\right) \\
d_{c, k}=\left(\begin{array}{ccccc}
-d_{R_{1}} & \cdots & 0 & 0 & 0 \\
\vdots & \ddots & \vdots & \vdots & \vdots \\
f_{k-1,1}^{k} & \cdots & -d_{R_{k-1}} & 0 & 0 \\
f_{k, 1}^{k} & \cdots & f_{k, k-1}^{k} & -d_{R_{k}} & 0 \\
f_{k, 1}^{k^{\prime}} & \cdots & f_{k, k-1}^{k^{\prime}} & f_{k, k}^{k^{\prime}} & d_{N_{0, k}}
\end{array}\right)
\end{gathered}
$$

Here

$$
f_{m, n}^{k}=\beta_{m} \circ \beta_{m-1}^{\prime} \cdots \beta_{n+1}^{\prime} \circ f_{n},
$$

for $1 \leq n<m \leq k$;

for $1 \leq n \leq k-1$; and

$$
f_{k, n}^{k^{\prime}}=\beta_{k}^{\prime} \circ \beta_{k-1}^{\prime} \cdots \beta_{n+1}^{\prime} \circ f_{n},
$$

$$
f_{k, k}^{k^{\prime}}=f_{k}
$$

Then $\left(D_{*}^{k}, d_{c, k}\right)$ is a chain complex.

Proof. We prove $d_{c, k} \circ d_{c, k}=0$ by using induction for $k$. If $k=1$, then $D_{*}^{1}=C_{*-1}\left(R_{1}\right) \oplus C_{*}\left(N_{0,1}\right)$ and

$$
d_{c, 1}=\left(\begin{array}{cc}
-d_{R_{1}} & 0 \\
f_{1} & d_{N_{0,1}}
\end{array}\right) .
$$

It is obviously a chain complex. Assume that $\left(D_{*}^{k}, d_{c, k}\right)$ is a chain complex, we want to show that $\left(D_{*}^{k+1}, d_{c, k+1}\right)$ is a chain complex. Notice that for $1 \leq n<$ $m \leq k$, we have

$$
f_{m, n}^{k+1}=f_{m, n}^{k}
$$

Therefore $d_{c, k}$ and $d_{c, k+1}$ can be expressed by block matrices:

and

$$
d_{c, k}=\left(\begin{array}{ll}
\tilde{d}_{k} & 0 \\
\tilde{f}_{k} & d_{N_{0, k}}
\end{array}\right)
$$

$$
d_{c, k+1}=\left(\begin{array}{ccc}
\tilde{d}_{k} & 0 & 0 \\
\beta_{k+1} \cdot \tilde{f}_{k}-d_{R_{k+1}} & 0 \\
\beta_{k+1}^{\prime} \cdot \tilde{f}_{k} & f_{k+1} & d_{N_{0, k+1}}
\end{array}\right)
$$


According to the assumption, we have the relations:

$$
\tilde{d}_{k} \circ \tilde{d}_{k}=0,
$$

and

$$
\tilde{f}_{k} \tilde{d}_{k}+d_{N_{0, k}} \tilde{f}_{k}=0 .
$$

Let $\beta_{k+1}$ and $\beta_{k+1}^{\prime}$ act on the above equality and using the formulas (2) and (3) in Lemma 4.1, we obtain

$$
\beta_{k+1} \cdot \tilde{f}_{k} \cdot \tilde{d}_{k}-d_{R_{k+1}} \cdot \beta_{k+1} \cdot \tilde{f}_{k}=0,
$$

and

$$
\beta_{k+1}^{\prime} \cdot \tilde{f}_{k} \cdot \tilde{d}_{k}+d_{N_{0, k+1}} \cdot \beta_{k+1}^{\prime} \cdot \tilde{f}_{k}+f_{k+1} \cdot \beta_{k+1} \tilde{f}_{k}=0 .
$$

combining the above two relations, the fact that $\tilde{d}_{k} \circ \tilde{d}_{k}=0$ and Lemma 4.2 , we can deduce $d_{c, k+1} \cdot d_{c, k+1}=0$.

Lemma 4.4 $\forall k=1, \cdots, s$, the cellular chain complex $\left(D_{*}^{k}, d_{c, k}\right)$ is chain isomorphic to $\left(C_{*}\left(X_{0}\right), d_{X_{0}}\right)$.

Proof. Firstly we will show the following map

$$
\begin{aligned}
& \left(\begin{array}{ccccc}
1 & \cdots & 0 & 0 & 0 \\
\vdots & \ddots & \vdots & \vdots & \vdots \\
0 & \cdots & 1 & 0 & 0 \\
0 & \cdots & 0 & \theta_{k+1} & 1
\end{array}\right)_{(k+1) \times(k+2)} \quad: C_{q-1}\left(R_{1}\right) \oplus C_{q-1}\left(R_{2}-\left(i_{1,+}\right)\left(R_{1}\right) \times \epsilon I^{1}\right) \oplus \cdots \\
& \oplus C_{q-1}\left(R_{k+1}-\cup_{j=1}^{k}\left(i_{j,+}\right) \times \epsilon I^{j}\right) \otimes C_{q}\left(N_{0, k+1}\right) \rightarrow \\
& C_{q-1}\left(R_{1}\right) \oplus C_{q-1}\left(R_{2}-\left(i_{1,+}\right)\left(R_{1}\right) \times \epsilon I^{1}\right) \oplus \cdots \oplus C_{q-1}\left(R_{k}-\cup_{j=1}^{k-1}\left(i_{j,+}\right)\right. \\
& \left.\times \epsilon I^{j}\right) \otimes C_{q}\left(N_{0, k}\right)
\end{aligned}
$$

is a chain map, i.e., it should satisfy the commutation relation:

$$
\left(\begin{array}{ccccc}
1 & \cdots & 0 & 0 & 0 \\
\vdots & \ddots & \vdots & \vdots & \vdots \\
0 & \cdots & 1 & 0 & 0 \\
0 & \cdots & 0 & \theta_{k+1} & 1
\end{array}\right) \circ d_{c, k+1}=d_{c, k} \circ\left(\begin{array}{ccccc}
1 & \cdots & 0 & 0 & 0 \\
\vdots & \ddots & \vdots & \vdots & \vdots \\
0 & \cdots & 1 & 0 & 0 \\
0 & \cdots & 0 & \theta_{k+1} & 1
\end{array}\right) \text {. }
$$

This is equivalent to the following equality:

$$
\left(\begin{array}{ccc}
\tilde{d}_{k} & 0 & 0 \\
\theta_{k+1} \beta_{k+1} \tilde{f}_{k}+\beta_{k+1}^{\prime} & \cdot \tilde{f}_{k}-\theta_{k+1} d_{R_{k+1}}+f_{k+1} & d_{N_{0, k+1}}
\end{array}\right)=\left(\begin{array}{ccc}
\tilde{d}_{k} & 0 & 0 \\
\tilde{f}_{k} d_{N_{0, k}} \theta_{k+1} & d_{N_{0, k}}
\end{array}\right),
$$


where $\tilde{f}_{k}, \tilde{d}_{k}$ are defined in Lemma 4.3 . Now this is true by Lemma 4.1 and the fact $\left.d_{N_{0, k}}\right|_{N_{0, k+1}}=d_{N_{0, k+1}}$. Thus we proved

$$
\left(\begin{array}{ccc}
I d & 0 & 0 \\
0 & \theta_{k+1} & 1
\end{array}\right)
$$

is a chain map. It is also an isomorphism, since it has an inverse chain map:

$$
\left(\begin{array}{cc}
I d & 0 \\
0 & \beta_{k+1} \\
0 & \beta_{k+1}^{\prime}
\end{array}\right) .
$$

Therefore we proved $\left(D_{*}^{k+1}, d_{c, k+1}\right)$ is chain isomorphic to $\left(D_{*}^{k}, d_{c, k}\right)$ for any $k=1, \cdots, s$. Note the $k=1$ case was already proved in Proposition 4.1.1 in [FJ2].

Define $\pi_{+}$as the monoid constructed by $\left(G_{+}, e\right)$ with the group action from $\pi$. Let $\rho_{e}: \pi_{1}(X) \rightarrow \pi$ be the extension of $\pi$ by the normal group $\pi_{1}(\bar{X})$. Since $\pi_{+}$is a monoid in $\pi$, the set $\rho_{e}^{-1}\left(\pi_{+}\right)$is also a monoid of $\pi_{1}(X)$. We denote $\rho_{e}^{-1}\left(\pi_{+}\right)$by $\pi_{1}(X)_{+} \cdot \mathbb{Z} \pi_{1}(X)_{+}$is a subring of $\mathbb{Z} \pi_{1}(X)$.

Tensor $\left(D_{*}^{k}, d_{c, k}\right)$ with the ring $\mathbb{Z} \pi_{1}(X)_{+}$, then we have the $\mathbb{Z} \pi_{1}(X)_{+}$-module chain complex $\left(\mathbb{Z} \pi_{1}(X)_{+} \otimes D_{*}^{k}, I \otimes d_{c, k}\right)$.

On the other hand, we can lift the related quantities (subcomplexes, or maps) to the universal covering space $\tilde{X}$ such that they become $\pi_{1}(X)_{+}$-equivariant. For instance, we can lift the $m-1$-dimensional polyhedra $\cup_{g \in \pi_{+}} g \cdot R_{i}$ to $\tilde{X}$ such that the obtained polyhedra $\tilde{R}_{i}$ is $\pi_{1}(X)_{+}$-equivariant. If $F$ is a map $\left(\beta_{k}, \beta_{k}^{\prime}, f_{k}\right.$, etc.), then the action of the lifted map $\tilde{F}$ is defined as: $\tilde{F}(g \cdot e)=g \cdot \tilde{F}(e)$. Thus we can get a $\mathbb{Z} \pi_{1}(X)_{+}$-module cellular chain complexes $\left(\tilde{D}_{*}^{k}, \tilde{d}_{c, k}\right)$, where

$$
\begin{aligned}
\tilde{D}_{q}^{k}:= & C_{q-1}\left(\tilde{R}_{1}\right) \oplus C_{q-1}\left(\tilde{R}_{2}-\left(\tilde{i}_{1,+}\right)\left(\tilde{R}_{1}\right) \times \epsilon I^{1}\right) \oplus \cdots \\
& \oplus C_{q-1}\left(\tilde{R}_{k}-\cup_{j=1}^{k-1}\left(\tilde{i}_{j,+}\right) \times \epsilon I^{j}\right) \oplus C_{q}\left(\tilde{N}_{0, k}\right)
\end{aligned}
$$

and $\tilde{d}_{c, k}$ is defined by the same form of the matrix (4.2), and the difference is that the entries of (4.2) are replaced by their lifting homomorphisms.

It is easy to see that the two $\mathbb{Z} \pi_{1}(X)_{+}$-module chain complexes $\left(\mathbb{Z} \pi_{1}(X)_{+} \otimes\right.$ $\left.D_{*}^{k}, I \otimes d_{c, k}\right)$ and $\left(\tilde{D}_{*}^{k}, \tilde{d}_{c, k}\right)$ are chain equivalent.

Let $\tilde{X}_{+}=\cup_{g \in \pi_{1}(X)_{+}} g \cdot \tilde{X}_{0}$. By Lemma 4.4, it is obvious that the following holds.

Proposition 4.5 The two $\mathbb{Z} \pi_{1}(X)_{+}$-module chain complexes $\left(C_{*}\left(\tilde{X}_{+}\right), d_{\tilde{X}_{+}}\right)$and $\left(\tilde{D}_{*}^{s}, \tilde{d}_{c, s}\right)$ are chain equivalent.

Monodromy representations and Novikov numbers In this part, we will 
generalize the crucial theorem, Theorem 4.2.1 in [FJ2] for an integral cocycle $\alpha$ to a higher rank cocycle $\alpha$. To get such a theorem, one needs also to study the monodromy representation and the evaluation representation as in [FJ2].

Since $\pi$ is a commutative group with rank $s$, we obtain a group homomorphism

$$
\rho_{\pi_{1}}: \pi_{1}(X) \stackrel{\rho_{e}}{\longrightarrow} \pi \stackrel{\rho_{A}}{\longrightarrow} \mathbb{Z}^{s} .
$$

Here $\rho_{A}\left(l_{1}^{a_{1}} \cdots l_{s}^{a_{s}}\right):=\left(a_{1}, \cdots, a_{s}\right)$. Thus we have a ring homomorphism

$$
\rho_{\pi_{1}}: \mathbb{Z}\left[\pi_{1}(X)\right] \longrightarrow \mathbb{Z}\left[\mathbb{Z}^{s}\right] .
$$

This ring homomorphism can induce another ring homomorphism

$$
\rho_{q}: \mathbb{Z}\left[\pi_{1}(X)\right] \rightarrow Q_{s}=\mathbb{Z}\left[t_{i}, t_{i}^{-1} ; i=1,2, \cdots, s\right]
$$

defined as, for $g=\sum z_{j}\left(l_{1}^{a_{j_{1}}} \cdot l_{s}^{a_{j_{s}}}\right) \in \mathbb{Z}[\pi]$,

$$
\rho_{q}(g)=\sum z_{j} t_{1}^{a_{j_{1}}} \cdots t_{s}^{a_{j_{s}}}
$$

In fact, $\rho_{q}$ is fully determined by the group $\pi$ and its representation. Restricting $\rho_{q}$ to the subring $\mathbb{Z}\left[\pi_{1}(X)_{+}\right]$, we can get a ring homomorphism

$$
\rho_{P}=\left.\rho_{q}\right|_{\mathbb{Z}\left[\pi_{1}(X)_{+}\right]}: \mathbb{Z}\left[\pi_{1}(X)_{+}\right] \longrightarrow P_{s}=\mathbb{Z}\left[t_{1}, \cdots, t_{s}\right]
$$

Let $\tilde{E}$ be a local system of free abelian groups on the compact polyhedron $X$, then $\tilde{E}$ is determined by its monodromy representation $\rho_{\tilde{E}}$ :

$$
\rho_{\tilde{E}}: \pi_{1}\left(X, x_{0}\right) \longrightarrow \operatorname{Aut}\left(\tilde{E}_{0}\right)=G L(k, \mathbb{Z})
$$

where $\tilde{E}_{0}$ is the fibre of the free abelian group at $x_{0}$ and $k=\operatorname{rank}\left(\tilde{E}_{0}\right)$. Let $E=\tilde{E} \otimes \mathbb{C}$, then $E$ is a complex flat vector bundle with the holonomy $\rho_{E}$

$$
\rho_{E}: \pi_{1}\left(X, x_{0}\right) \longrightarrow G L(k ; \mathbb{Z}) \otimes \mathbb{C}
$$

Now the tensor product of the representations $\rho_{q} \otimes \rho_{\tilde{E}}$ gives a representation of a $\mathbb{Z}\left[\pi_{1}(X)\right]$-ring to the linear space $\left(Q_{s}\right)^{k}$, where $Q_{s}$ is the polynomial space with $s$ variables over $\mathbb{Z}$.

Since $\rho_{\tilde{E}}$ is an anti-homomorphism, i.e., $\forall g, g^{\prime} \in \mathbb{Z}\left[\pi_{1}(X)\right], \rho_{E}\left(g \cdot g^{\prime}\right)=\rho_{E}\left(g^{\prime}\right)$. $\rho_{E}(g)$, hence $\rho_{P} \otimes \rho_{\tilde{E}}$ gives a right $\mathbb{Z}\left[\pi_{1}(X)\right]_{+}$-module structure on $P_{s}^{k}$. With the $P_{s}$-module structure of itself, $P_{s}^{k}$ becomes a $\left(P_{s}, \mathbb{Z}\left[\pi_{1}(X)_{+}\right]\right)$-bimodule.

Define $D_{*}=P_{s}^{k} \otimes_{\mathbb{Z}\left[\pi_{1}(X)\right]_{+}} C_{*}\left(\tilde{X}_{+}\right)$, then $D_{*}$ is a $P_{s}$-module chain complex.

Evaluation representations Take any complex $s$-vector $a=\left(a_{1}, \cdots, a_{s}\right) \in$ $\mathbb{C}^{s}$. The complex number field $\mathbb{C}$ can be given a $P_{s}$-module structure, whose module structure is provided by the action: for a polynomial $P\left(t_{1}, \cdots, t_{s}\right), P\left(t_{1}, \cdots, t_{s}\right)$. $x=P\left(a_{1}, \cdots, a_{s}\right) \cdot x=P(a) \cdot x$ for $x \in \mathbb{C}$. We denote the $P_{s}$-module of $\mathbb{C}$ evaluated at $t=a$ by $\mathbb{C}_{a}$. Similarly for any $a \in\left(\mathbb{C}^{*}\right)^{s}, \mathbb{C}$ can be viewed as a $Q_{s}$-module. 
If $p$ is a prime number, then the field $\mathbb{Z}_{p}$ also has a $P_{s}$-module structure which is given by the evaluation at $t=0$. We consider the complexes $\mathbb{C}_{a} \otimes_{P_{s}} D_{*}$ and $\mathbb{Z}_{p} \otimes_{P_{s}} D_{*}$. The following theorem is the generalization of Theorem 4.2.1 in [FJ2] for higher rank $\alpha$.

Theorem 4.6 Assume rank $=s \geq 1$. Let $D_{*}=P_{s}^{k} \otimes_{\mathbb{Z}\left[\pi_{1}(X)_{+}\right]} C_{*}\left(\tilde{X}_{+}\right)$be defined by the above argument. We have

(1) For any nonzero complex vector $a \in\left(\mathbb{C}^{*}\right)^{s}$, the homology $H_{*}\left(\mathbb{C}_{a} \otimes_{P_{s}} D_{*}\right)$ is isomorphic to $H_{*}\left(X, a^{\alpha} \otimes E\right)$, which is viewed as the homology of the presheaf $a^{\alpha} \otimes E$ on $X$, where the presheaf $a^{\alpha}$ on $X$ is given by the monodromy representation $\rho_{P}: \mathbb{Z}\left[\pi_{1}(X)_{+}\right] \rightarrow P_{s}(a)=\mathbb{Z}\left[a_{1}, \cdots, a_{s}\right]$.

(2) Let $p$ be a prime number and let $\mathbb{Z}_{p}$ have the $P_{s}$-module structure which is provided by the evaluation at $t=\left(t_{1}, \cdots, t_{s}\right)=0$. Then the homology $H_{*}\left(\mathbb{Z}_{p} \otimes_{P_{s}} D_{*}\right)$ is isomorphic to $H_{*}\left(X_{0, s}, \cup_{j=1}^{s}\left(i_{j,+}\right)\left(R_{j}\right) ; \mathbb{Z}_{p} \otimes P_{\pi}^{*} \tilde{E}\right)$, where $\tilde{E}$ is a local system on $X$ and $P_{\pi}^{*} E$ is the pull-back local system on $X_{0, s}$ by the projection $P_{\pi}: \bar{X} \longrightarrow X$.

(3) $H_{*}\left(C_{0} \otimes_{P_{s}} D_{*}\right)$ is isomorphic to $H_{*}\left(X_{0, s}, \cup_{j=1}^{s}\left(i_{j,+}\right)\left(R_{j}\right) ; P_{\pi}^{*} E\right)$.

Proof. Since the proof of (3) is the same as that of (2), we only give the proofs of (1) and (2).

(1). Since the $\mathbb{Z}\left[\pi_{1}(X)_{+}\right]$-basis of $C_{*}\left(\tilde{X}_{+}\right)$is finite, all the complexes related to $C_{*}\left(\tilde{X}_{+}\right)$are finitely generated, and hence all the homology groups are finitely generated.

For $a \in\left(C^{*}\right)^{s}$, we have the isomorphism

$$
\begin{aligned}
\mathbb{C}_{a} \otimes_{P_{s}} D^{*} & \cong \mathbb{C}_{a} \otimes_{P_{s}}\left(\left(P_{s}\right)^{k} \otimes_{\mathbb{Z}\left[\pi_{1}(X)_{+}\right]} C_{*}\left(\tilde{X}_{+}\right)\right) \\
& \cong\left(\mathbb{C}_{a} \otimes_{Q(s)}\left(Q_{s}\right)^{k}\right) \otimes_{\mathbb{Z}\left[\pi_{1}(X)\right]}\left(\mathbb{Z}\left[\pi_{1}(X)\right] \otimes_{\mathbb{Z}\left[\pi_{1}(X)_{+}\right]} C_{*}\left(\tilde{X}_{+}\right)\right) \\
& \cong\left(\mathbb{C}_{a} \otimes_{\mathbb{Z}} \mathbb{Z}^{k}\right) \otimes_{\mathbb{Z}\left[\pi_{1}(X)\right]} C_{*}(\tilde{X}) \\
& \cong \mathbb{C}^{k} \otimes_{\mathbb{Z}\left[\pi_{1}(X)\right]} C_{*}(\tilde{X}) .
\end{aligned}
$$

Here the representation of $\mathbb{Z}\left[\pi_{1}(X)\right]$ is given by

$$
g \longrightarrow \rho_{P(a)}(g) \otimes \rho_{E}(g) .
$$

Hence the homology of $\mathbb{C}^{k} \otimes_{\mathbb{Z}\left[\pi_{1}(X)\right]} C_{*}(\tilde{X})$ is the same as the homology of the presheaf $a^{\pi} \otimes E$ on $X$ that corresponds to the flat vector bundle produced by the above holonomy representation. (1) is proved. 
$(2)$

$$
\mathbb{Z}_{p} \otimes_{P_{s}} D^{*} \cong \mathbb{Z}_{p} \otimes_{P_{s}}\left(P_{s}^{k} \otimes_{\mathbb{Z}\left[\pi_{1}(X)_{+}\right]} C_{*}\left(\tilde{X}_{+}\right)\right) \cong \mathbb{Z}_{p}^{k} \otimes_{\mathbb{Z}\left[\pi_{1}(X)_{+}\right]} C_{*}\left(\tilde{X}_{+}\right) .
$$

Here the representation of $\mathbb{Z}\left[\pi_{1}(X)_{+}\right]$on $\mathbb{Z}_{p}^{k}$ is

$$
g \longrightarrow \rho_{P(0)}(g) \otimes \rho_{E}(g) .
$$

Since $\rho_{P(0)}(g)=\rho_{P(0)} \cdot \rho_{A} \rho_{e}(g)$, except in the case that $g \in \mathbb{Z}\left[\pi_{1}(X)_{+}\right]$satisfies $\rho_{e}(g)=1$, the evaluation representation will make the final representation vanish. Hence (4.3) becomes

$$
\begin{array}{r}
g \longrightarrow \rho_{E}(g), \text { if } \rho_{e}(g)=1 \\
g \longrightarrow 0, \text { if } \rho_{e}(g) \neq 1 .
\end{array}
$$

By Proposition 4.5 , in order to prove $(2)$, we need to prove that $\mathbb{Z}_{p}^{k} \otimes_{\mathbb{Z}\left[\pi_{1}(X)_{+}\right]} \tilde{D}_{*}^{s}$ is equivalent to $\left(\mathbb{Z}_{p} \otimes P_{\pi}^{*} \tilde{E}\right) \otimes C_{*}\left(X_{0, s}, \cup_{j=1}^{s}\left(i_{j,+}\right)\left(R_{j}\right)\right)$. Now we have

$$
\mathbb{Z}_{p}^{k} \otimes_{\mathbb{Z}\left[\pi_{1}(X)_{+}\right]} \tilde{D}_{q}^{s}=\left(\mathbb{Z}_{p}^{k} \otimes_{\mathbb{Z}\left[\pi_{1}(X)_{+}\right]} C_{q-1}\left(\tilde{R}_{1}\right)\right) \oplus \cdots\left(\mathbb{Z}_{p}^{k} \otimes_{\mathbb{Z}\left[\pi_{1}(X)_{+}\right]} \oplus C_{q}\left(\tilde{N}_{0, s}\right)\right) .
$$

If we take $\tilde{E} \otimes C_{*}\left(R_{t}-\cup_{j=1}^{t-1}\left(i_{j,+}\right)\left(R_{j}\right) \times \epsilon I^{j}, N^{+}\right)$as the complexes with twisted coefficients $\tilde{E}$, then $\mathbb{Z}_{p}^{k} \otimes_{\mathbb{Z}\left[\pi_{1}(X)_{+}\right]} C_{*}\left(\tilde{R}_{t}-\cup_{j=1}^{t-1}\left(\tilde{i}_{j,+}\right)\left(\tilde{R}_{j}\right) \times \epsilon I^{j}\right) \cong \mathbb{Z}_{p} \otimes\left(\tilde{E} \otimes C_{*}\left(R_{t}-\right.\right.$ $\left.\cup_{j=1}^{t-1}\left(i_{j,+}\right)\left(R_{j}\right) \times \epsilon I^{j}, N^{+}\right)$) (Notice that the evaluation map at $t=0$ makes the cell lying in $N^{+}$vanishing $)$. Similarly $\mathbb{Z}_{p}^{k} \otimes_{\mathbb{Z}\left[\pi_{1}(X)_{+}\right]} C_{*}\left(\tilde{N}_{0, t}\right) \cong \mathbb{Z}_{p} \otimes\left(P_{\pi}^{*} \tilde{E} \otimes\right.$ $C_{*}\left(X_{0, t}\right)$ ). Therefore

$$
\mathbb{Z}_{p}^{k} \otimes_{\mathbb{Z}\left[\pi_{1}(X)_{+}\right]} \tilde{D}_{*}^{s} \cong\left(\mathbb{Z}_{p} \otimes\left(\tilde{E} \otimes C_{*-1}\left(R_{1}, N^{+}\right)\right)\right) \oplus \cdots \oplus\left(\mathbb{Z}_{p} \otimes\left(P_{\pi}^{*} \tilde{E} \otimes C_{*}\left(X_{0, s}\right)\right)\right.
$$

Denote this complex by $D_{*}^{s, 0}$. Its differential becomes

$$
d_{c}^{s, 0}=\left(\begin{array}{ccccc}
-d_{R_{1}} & \cdots & 0 & 0 & 0 \\
\vdots & \ddots & \vdots & \vdots & \vdots \\
i_{s-1,1}^{s} & \cdots & -d_{R_{s-1}} & 0 & 0 \\
i_{s, 1}^{s} & \cdots & i_{s, s-1}^{s} & -d_{R_{s}} & 0 \\
i_{s, 1}^{s} & \cdots & i_{s, s-1}^{s \prime} & i_{s, s}^{s \prime} & d_{N_{0, s}}
\end{array}\right)
$$

Here

$$
i_{m, n}^{s}=-\beta_{m} \circ \beta_{m-1}^{\prime} \cdots \beta_{n+1}^{\prime} \circ i_{n,+}
$$

for $1 \leq n<m \leq s$,

$$
i_{s, n}^{s \prime}=-\beta_{s}^{\prime} \circ \beta_{s-1}^{\prime} \cdots \beta_{n+1}^{\prime} \circ i_{n,+},
$$

for $1 \leq n \leq s-1$, and

$$
i_{s, s}^{s \prime}=-i_{s,+}
$$

Now for convenience, we assume the coefficient ring is the integer ring. 
Define maps

$$
\begin{aligned}
& I_{1}:=\left(i_{2,1}^{s}, \cdots, i_{s, 1}^{s}, i_{s, 1}^{s^{\prime}}\right): C_{*}\left(R_{1}, N^{+}\right) \rightarrow \\
& D_{*}^{s, 1}:=C_{*-1}\left(R_{2}-\left(i_{1,+}\right)\left(R_{1}\right) \times \epsilon I^{1}, N^{+}\right) \oplus \cdots \oplus C_{*}\left(X_{0, s}\right),
\end{aligned}
$$

and

$P_{1}:=D_{*}^{s, 1} \rightarrow \bar{D}_{*}^{s, 1}:=C_{*-1}\left(R_{2}-\left(i_{1,+}\right)\left(R_{1}\right) \times I^{1}, R_{1} \cup N^{+}\right) \oplus \cdots \oplus C_{*}\left(X_{0, s}, R_{1}\right)$

to be the quotient map. Let $d_{c}^{s, 1}$ be the matrix obtained from (4.4) by deleting the first row and the first column. We have $d_{c}^{s, 1} \circ d_{c}^{s, 1}=0$. Hence $\left(D_{*}^{s, 1}, d_{c}^{s, 1}\right)$ is a chain complex. It induces the quotient chain complex $\left(\bar{D}^{s, 1}, \bar{d}_{c}^{s, 1}\right)$. The following is a short exact sequence.

$$
0 \rightarrow C_{*}\left(R_{1}\right) \stackrel{I_{1}}{\longrightarrow} D_{*}^{s, 1} \stackrel{P_{1}}{\longrightarrow} \bar{D}_{*}^{s, 1} \rightarrow 0
$$

To see this, we have to show the following three points:

(i) $P_{1} \circ I_{1}=0$

(ii) $\operatorname{ker} P_{1} \subset \operatorname{im} I_{1}$

(iii) $I_{1}, P_{1}$ are chain maps.

(i) is obvious. Assume $P_{1}\left(\alpha_{2}, \cdots, \alpha_{s}, \alpha_{s}^{\prime}\right)=0$, i.e., $\forall j=2, \cdots, s$,

$$
\alpha_{j} \in R_{1} \cap R_{j}-\cup_{t=1}^{j-1} R_{t} \times \epsilon I^{t}-N^{+}
$$

and

$$
\alpha_{s}^{\prime} \in X_{0, s}-\cup_{j=2}^{s} R_{j}
$$

Let

$$
\alpha=-\left(\sum_{j=2}^{s} \theta_{j}\left(\alpha_{j}\right)+\alpha_{s}^{\prime}\right) .
$$

Then we have $I_{1}(\alpha)=\left(\alpha_{2}, \cdots, \alpha_{s}, \alpha_{s}^{\prime}\right)$. Hence $(i i)$ is proved. To prove (iii), one need only to check the commutative diagram:

$$
\begin{aligned}
& C_{q}\left(R_{1}\right) \stackrel{I_{1}}{\longrightarrow} D_{q}^{s, 1} \\
& \downarrow d_{R_{1}} \quad \downarrow d_{c}^{s, 1} \\
& C_{q-1}\left(R_{1}\right) \stackrel{I_{1}}{\longrightarrow} D_{q-1}^{s, 1}
\end{aligned}
$$

i.e., check the equality: $d_{c}^{s, 1} \cdot I_{1}=I_{1} \cdot d_{R_{1}}$. This holds in view of the relation $d^{s, 0} \cdot d^{s, 0}=0$. Hence $($ iii $)$ is proved.

From the short exact sequence (4.5), we obtain the long exact sequence:

$$
\begin{aligned}
& \cdots \rightarrow H_{q}\left(R_{1}\right) \stackrel{I_{1}}{\longrightarrow} H_{q}\left(D_{*}^{s, 1}\right) \rightarrow H_{q}\left(\bar{D}_{*}^{s, 1}\right) \rightarrow \\
& \rightarrow H_{q-1}\left(R_{1}\right) \stackrel{I_{1}}{\longrightarrow} H_{q-1}\left(D_{*}^{s, 1}\right) \rightarrow \cdots
\end{aligned}
$$


Similarly, from the short exact sequence

$$
0 \rightarrow C_{*}\left(D_{*}^{s, 1}\right) \rightarrow C_{*}\left(D_{*}^{s, 0}\right) \rightarrow\left(C_{*-1}\left(R_{1}\right),-d_{R_{1}}\right) \rightarrow 0
$$

we get the long exact sequence

$$
\cdots \rightarrow H_{q}\left(R_{1}\right) \stackrel{I_{1}}{\longrightarrow} H_{q}\left(D_{*}^{s, 1}\right) \rightarrow H_{q}\left(D_{*}^{s, 0}\right) \rightarrow H_{q-1}\left(R_{1}\right) \stackrel{I_{1}}{\longrightarrow} H_{q-1}\left(D_{*}^{s, 1}\right) \rightarrow \cdots
$$

The two long exact sequences yield the following two short exact sequences

$$
\begin{aligned}
0 \rightarrow \operatorname{Coker}\left(I_{1}: H_{q}\left(R_{1}\right) \rightarrow H_{q}\left(D_{*}^{s, 1}\right)\right) & \rightarrow H_{q}\left(\bar{D}_{*}^{s, 1}\right) \rightarrow \\
& \rightarrow \operatorname{Ker}\left(I_{1}: H_{q-1}\left(R_{1}\right) \rightarrow H_{q-1}\left(D_{*}^{s, 1}\right)\right) \rightarrow 0
\end{aligned}
$$

and

$$
\begin{aligned}
0 \rightarrow \operatorname{Coker}\left(I_{1}: H_{q}\left(R_{1}\right) \rightarrow H_{q}\left(D_{*}^{s, 1}\right)\right) & \rightarrow H_{q}\left(D_{*}^{s, 0}\right) \rightarrow \\
& \rightarrow \operatorname{Ker}\left(I_{1}: H_{q-1}\left(R_{1}\right) \rightarrow H_{q-1}\left(D_{*}^{s, 1}\right)\right) \rightarrow 0
\end{aligned}
$$

Using the Five-Lemma, we get for any $q \geq 0$

$$
H_{q}\left(\bar{D}_{*}^{s, 1}\right) \cong H_{q}\left(D_{*}^{s, 0}\right) .
$$

Continuing our operation, finally we get

$$
H_{q}\left(D_{*}^{s, 0}\right) \cong H_{q}\left(\bar{D}_{*}^{s, s}\right)=H_{q}\left(X_{0, s}, \cup_{j=1}^{s} R_{j}\right) .
$$

Hence we finally proved the second conclusion of this theorem.

Novikov numbers In Theorem 4.4, we have considered the complex $D_{*}=P_{s}^{k}$ $\otimes_{\mathbb{Z}\left[\pi_{1}(X)_{+}\right]} C_{*}\left(\tilde{X}_{+}\right)$. In this part, we always let the vector bundle $E$ that appears in Theorem 4.6 be a trivial line bundle. Then the complex $D_{*}$ there has the form $D_{*}=P_{s} \otimes_{\mathbb{Z}\left[\pi_{1}(X)_{+}\right]} C_{*}\left(\tilde{X}_{+}\right)$. Since the representation of $\mathbb{Z}\left[\pi_{1}(X)_{+}\right]$in $P_{s}$ is completely determined by the cohomology class $[\alpha]$, we denote the homology group $H_{*}\left(\mathbb{C}_{a} \otimes_{P_{s}} D_{*}\right)$ as $H_{*}\left(X, a^{\alpha}\right)$, or in other words, view the homology group $H_{*}\left(\mathbb{C}_{a} \otimes_{P_{s}} D_{*}\right)$ as the homology group of the presheaf $a^{\alpha}$ on $X$ which is given by the monodromy representation $\rho_{P}: \mathbb{Z}\left[\pi_{1}(X)_{+}\right] \rightarrow P_{s}=\mathbb{Z}\left[t_{1}, \cdots, t_{s}\right]$.

The following is essentially given in [No3].

Proposition 4.7 Let $X$ be a compact polyhedron. Define a function for fixed $i$ to be

$$
a \in\left(\mathbb{C}^{*}\right)^{s} \longrightarrow \operatorname{dim}_{\mathbb{C}} H_{i}\left(X, a^{\alpha}\right),
$$

then it has the following properties:

(1) It is generically constant, more precisely, except on a proper algebraic subvariety $\mathcal{L}$ in $\left(\mathbb{C}^{*}\right)^{s}$, the dimension $\operatorname{dim}_{\mathbb{C}} H_{i}\left(X, a^{\alpha}\right)$ is constant and this constant is just the Novikov number $b_{i}([\alpha])$ we defined above.

(2) For any point $\hat{a} \in \mathcal{L}$,

$$
\operatorname{dim}_{\mathbb{C}} H_{i}\left(X, \hat{a}^{\alpha}\right)>b_{i}([\alpha])
$$


Definition $b_{i}([\alpha]):=\operatorname{rank}\left(H_{i}\left(D_{*}\right)\right)$ for $i=0,1, \cdots, m$ are called the Novikov numbers.

Remark If $s=1$ and $X$ is a closed manifold, then it was proved in [Fa5] that the Novikov number we defined here is the same as the Novikov number which is defined as the dimension of the homology group of the Novikov complex.

\section{Novikov-Morse type inequalities for higher rank $\alpha$}

Theorem 4.6 is crucial in the proof of Novikov-Morse type inequalities. As long as we obtain theorem 4.6 for flows carrying a higher rank cocycle $\alpha$, we can follow the same line as in the proof in [FJ2] to get the expected analogous inequalities. We omit the similar proof as in section 4.4 and 4.5 of [FJ2], and only list the results.

Ideals Let $P_{s}=\mathbb{Z}\left[t_{1}, \cdots, t_{s}\right]$ be the polynomial ring with $s$ variables over the integers $\mathbb{Z}$. Let $\left\langle t_{1}, \cdots, t_{s}\right\rangle$ be the ideal generated by $t_{1}, \cdots, t_{s}$. Define $I=\left\{t \in \mathbb{C}^{s} ; 1+\left\langle t_{1}, \cdots, t_{s}\right\rangle=0\right\}$. Let $a \in\left(\mathbb{C}^{*}\right)^{s}$ be not in $I$. Define $I_{a}$ to be the prime ideal in the polynomial ring $P_{s}$ consisting of the polynomials vanishing at $a$. By the choice of $a$, the free terms of all the polynomials $f(t) \in I_{a}$ are divisible by some prime number $p$. Therefore we obtain

$$
I_{a} \subset I_{p}=\langle p\rangle+\left\langle t_{1}, \cdots, t_{s}\right\rangle
$$

Theorem 5.1 Let $X$ be a compact polyhedron with a metric d. Let $\alpha$ be an continuous cocycle and $v$ be a generalized $\alpha$-flow w.r.t. an isolated invariant set $\mathcal{A}=\left\{A_{1}, \cdots, A_{n}\right\}$. Let $\tilde{E}$ be a local system of free abelian groups and let $E=\mathbb{C} \otimes \tilde{E}$. If $a \in \mathbb{C}^{*}$ and $a \notin I=1+\left\langle t_{1}, \cdots, t_{s}\right\rangle \subset P_{s}$, then there is a prime number $p$ relative to a such that

$$
\begin{aligned}
& \sum_{A_{i} \in \mathcal{A}} p\left(h\left(A_{i}\right) ; t, \mathbb{Z}_{p} \otimes J_{A_{i}}^{*} E\right)=p\left(X ; t, a^{\alpha} \otimes E\right) \\
& +(1+t) Q_{1}\left(I_{a}, I_{p} ; t\right)+(1+t) Q_{2}\left(I_{p} ; t\right)
\end{aligned}
$$

where $J_{A_{i}}$ is the inclusion map from the isolating neighborhood of an isolated invariant set $A_{i}$ to $X$, and $Q_{1}\left(I_{a}, I_{p} ; t\right)$ and $Q_{2}\left(I_{p} ; t\right)$ are all polynomials with nonnegative integer coefficients.

Corollary 5.2 (Euler-Poincaré formula) Under the hypothesis of Theorem 5.1 ,

$$
\sum_{A \in \mathcal{A}} p\left(h(A) ;-1, \mathbb{Z}_{p} \otimes J_{A}^{*} E\right)=p\left(X,-1, a^{\alpha} \otimes E\right)
$$


In particular, if $E$ is a trivial line bundle, then

$$
\sum_{A \in \mathcal{A}} p\left(h(A) ;-1, \mathbb{Z}_{p}\right)=\chi(X)
$$

for any prime number $p$. Here $\chi(X)$ is the Euler characteristic number of the compact polyhedron.

As explained in [FJ2], if the cocycle $\alpha$ is a trivial cocycle, then the above Novikov-Morse type inequalities will induce the Conley-Morse type inequalities given in [CZ]. If $\omega$ is a closed 1-form with higher rank, then Theorem 5.1 generalizes the rank one case which is proved in [FJ2]. We have

Theorem 5.3 Let $\omega$ be a closed 1-form with critical set $\mathcal{A}$ consisting of $f$ nitely many connected components. Each component is assumed to be Lipschitz submanifold. Let $\tilde{E}$ be a local system of free abelian groups and $E=\mathbb{C} \otimes \tilde{E}$. Assume that $a \in \mathbb{C}^{*}$ and $a \notin I=1+\left\langle t_{1}, \cdots, t_{s}\right\rangle \subset P_{s}$. Then there is a prime $p$ such that

$$
\begin{aligned}
& \sum_{A \in \mathcal{A}} p\left(h(A) ; t, \mathbb{Z}_{p} \otimes J_{A}^{*} E\right) \\
& =p\left(X, t ; a^{\omega} \otimes E\right)+(1+t) Q(E, t)
\end{aligned}
$$

where $Q(E, t)$ is a polynomial with nonnegative coefficients.

If the closed 1-form $\omega$ in Theorem 5.3 has only non-degenerate isolated zero points or Bott type non-degenerate zero locus, then the above theorem reduces to the theorems proven by M. Farber [Fa1, Fa2](The reader can also see [FJ2] for a complete description of those cases).

Corollary 5.4 (Classical Novikov inequality) Let $X$ be an oriented closed smooth manifold and $\omega$ a Morse closed 1-form. Then the numbers $c_{j}(\omega)$ of zeros of $\omega$ having index $j$ satisfy

$$
\begin{aligned}
c_{j}(\omega) & \geq b_{j}([\omega]) \\
\sum_{i=0}^{j}(-1)^{i} c_{j-i}(\omega) & \geq \sum_{i=0}^{j}(-1)^{i} b_{j-i}([\omega])
\end{aligned}
$$

for $j=0,1, \cdots, m$.

Theorem 5.5 Let $v$ be an $\alpha$-Morse-Smale flow w.r.t. $\mathcal{A}=\left\{A_{n}, \cdots, A_{1}\right\}$. Let $c_{j}$ be the number of hyperbolic fixed points with index $j, a_{j}$ be the number of 
the hyperbolic periodic orbits with index $j$, and $\mu_{j}=c_{j}+a_{j}+a_{j+1}$. Then

$$
\begin{aligned}
\mu_{j} & \geq b_{j}([\alpha]) \\
\sum_{i=0}^{j}(-1)^{j-i} \mu_{i} & \geq \sum_{i=0}^{j}(-1)^{j-i} b_{j}([\alpha])
\end{aligned}
$$

for $j=0,1, \cdots, m$.

Theorem 5.6 (Vanishing theorem) Let $X$ be a compact polyhedron with a metric $d$. If there exists a flow carrying a cohomology class $[\alpha]$ on $X$, then

$$
b_{i}([\alpha])=0, \quad \forall i=0,1, \cdots, m .
$$

Example 5.1 Let $f: X \rightarrow X$ be a homeomorphism from the compact polyhedron $X$ to itself, then we can define the mapping torus of $f$ :

$$
T_{f}:=\{(x, t) \mid x \in X, t \in[0.1]\} /(x, 0) \sim(f(x), 1) .
$$

Then the natural projection $\pi: T_{f} \rightarrow S^{1}$ provides a nontrivial cocycle $\alpha=J(\pi)$ : $X \times X \rightarrow \mathbb{R}$ (where $J$ is defined in proposition 3.1.1 of [FJ2]). Define the flow carrying the cohomology class $\alpha$ as: for $0 \leq s<1$, and $\forall t \in \mathbb{R}$,

$$
[x, s] \cdot t:=\left[f^{\llbracket s+t \rrbracket}(x),(s+t)\right],
$$

where $\llbracket \cdot \rrbracket$ is the function taking the integer part and $(\cdot)$ is the function taking the decimal part. According to Theorem 5.6, we have

$$
b_{i}([\alpha])=0, \forall i .
$$

\section{REFERENCES}

[BF] M. Braverman, M. Farber, Novikov type inequalities for differential forms with non-isolated zeros, Math. Proc. Cambridge Philos. Soc. 122 (1997), no. 2, 357-375.

[Ch] R. Churchill, Invariant sets which carry cohomology. J. Diff. Equ. 13(1973), 523-550

[Co] C. C. Conley, Isolated invariant sets and the Morse index, CBMS Reg. Conf. Series in Math. 38, 1978, A. M. S., Providence, R. I.

[CZ] C. C. Conley, E. Zehnder, Morse-type index theory for flows and periodic solutions for Hamiltonian equations, Comm. Pure and Appl. Math., Vol 37(1984), 207-253

[FJ1] H. Fan, and J. Jost, Novikov Morse theory for dynamical systems, (preliminary version)math.DS/0010295, 2000

[FJ2] H. Fan, and J. Jost, Novikov Morse theory for dynamical systems, Calc. Var. 17, 2973(2002).

[Fa1] M. Farber, Counting zeros of closed 1-forms, Topology, ergodic theory, real algebraic geometry, 95-107, Amer. Math. Soc. Transl. Ser. 2, 202, Amer. Math. Soc., Providence, RI, 2001.

[Fa2] Morse-Novikov critical point theory, Cohn localization and Dirichlet units, Commun. Contemp. Math. 1 (1999), no. 4, 467-495.

[Fa3] Z Zeroes of closed 1-forms, homoclinic orbits and Lusternik-Schnirelman theory, "Topological Methods in Nonlinear Analysis", 19(2002), 123-152/math.DG/0106046 
[Fa4] Lusternik-Schinrelman Theory and Dynamics, in: "Lusternik-Schinrelmann Category and Related Topics", O. Cornea et al. editors, Contemporary Mathematics, Vol. 316(2002).

[Fa5] M. Farber, Exactness of the Novikov inequalities, Func. Anal.Appl. 19(1985), 40-48

[FKLZ] M. Farber, T. Kappeler, J. Latschev and E. Zehnder, Lyapunov 1-forms for flows, arXiv:math.DS/0210473 v2 10 Feb 2003

[FR] M.Farber and A.A.Ranicki, The Morse-Novikov theory of circle valued functions and noncommutative localization, Proc. 1998 Moscow Conference for the 60th Birthday of S.P.Novikov, Tr. Mat. Inst. Steklova 225(1999) 381-388

[Fo1] R. Forman, Morse theory for cell complexes. Adv. Math. 134 (1998), no. 1, 90-145.

[Fo2] _ Combinatorial Novikov-Morse theory. Internat. J. Math. 13 (2002), no. 4, 333-368

[Fo3] , Discrete Morse theory and the cohomology ring. Trans. Amer. Math. Soc. 354 (2002), no. $12,5063-5085$ (electronic).

[No1] S. P. Novikov, Multivalued functions and functionals. An analog of Morse theory, Dokl. Akad. Nauk SSSR, 260, No.1(1981) 31-35

[No2] _ The Hamiltonian formalism and a multivalued analog of Morse theory, Usp. Mat. Nauk, 37, No.5 (1982) 3-49

[No3] _ Bloch homology, critical points of functions and closed 1-forms, Soviet. Math. Dokl. 33(1986) 551-555

[Pa] A. Pajitnov, Morse theory of closed 1-forms, LNM. 1474, Springer, Berlin, 1991

[Ra] A.A. Ranicki, The algebraic construction of the Novikov complex, e-print http://arXiv.org/abs/math/AT/9903090

[Sm] S. Smale, Morse inequalities for a dynamical system, Bull. A.M.S.66, (1960) 43-49

[Sp] E. Spanier, Algebraic Topology, McGraw-Hill, New York, 1966

Huijun Fan

School of mathematical sciences

Peking University

100871, Beijing

P.R. China

and

Max-Planck-Institute for

Mathematics in the Sciences

Inselstr. 22-26

04103 Leipzig

Germany

E-mail: fanhj@math.pku.edu.cn

Jürgen Jost

Max-Planck-Institute for

Mathematics in the Sciences

Inselstr. 22-26

04103 Leipzig

Germany

E-mail: jjost@mis.mpg.de 